\title{
Backbone Torsion Angle Determination Using Proton Detected Magic-Angle Spinning Nuclear Magnetic Resonance
}

Kai Xue, ${ }^{+a^{\star}}$ Evgeny Nimerovsky, ${ }^{+a}$ Kumar A. Tekwani Movellan, ${ }^{a, \# ~ S t e f a n ~ B e c k e r, ~}{ }^{a}$ Loren B. Andreas ${ }^{\text {* }}$

a Max Planck Institute for Biophysical Chemistry, Department of NMR Based Structural Biology, Am Fassberg. 11, Goettingen, Germany

\#Current Address: Department of Chemistry and Biochemistry, University of Delaware, Newark, DE 19716, United States

To whom correspondence should be addressed: kaxu@nmr.mpipc.mpg.de land@nmr.mpipc.mpg.de

+KX and EN contribute equally to this work 
Table of Contents

Dipolar Coupling Determination ................................................................................. 2

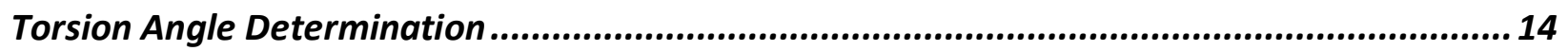

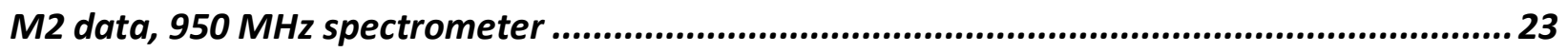

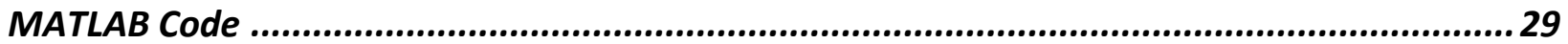

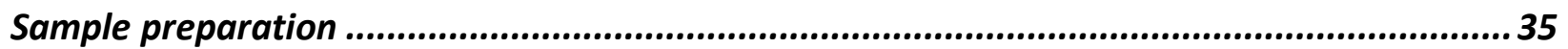

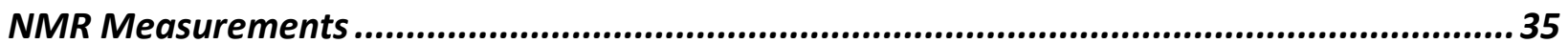

\section{Dipolar Coupling Determination}

MODER5 consists of two $90^{\circ}$ pulses with phases $36^{\circ}$ and $164.2^{\circ}$ as described in Ref. ${ }^{1}$. In an ideal case, the frequency of the MODER5 oscillations depends only on the dipolar coupling value, $v_{D}$. To obtain dipolar values we compare the normalized experimental MODERN signal, $\bar{S}_{\text {exp }}\left(t_{\text {exp }}\right)=S_{\text {exp }}\left(t_{\text {mix }}\right) / S_{\text {exp }}(0)$ with the normalized simulated, $\bar{S}_{\text {sim }}\left(v_{D} ; t_{\text {mix }}\right)=S_{\text {sim }}\left(v_{D} ; t_{\text {mix }}\right) /$ $S_{\text {sim }}(0)$ and minimize the value of the residual, $\chi_{v}^{2}$ :

$\chi_{v}^{2}=\left[\operatorname{sum}\left[\left(\bar{S}_{\text {exp }}\left(t_{\text {mix }}\right)-\bar{S}_{\text {sim }}\left(v_{D} ; t_{\text {mix }}\right)\right) / \text { error }\right]^{2}\right] /(v-1), \quad$ Eqn. $(\mathrm{S} 1)$

where, $v$ is the number of the experimental points; error - is the uncertainty in the peak amplitude, estimated from the RMSD of a noise region of the spectrum (the same region for all spectra in one series). Peak intensities were analyzed using software PINT (Ref. ${ }^{2}$ )

The MODERN signal is influenced by experimental imperfections, for example, rf-field inhomogeneity. Obtaining reasonable fits required modeling a distribution of flip angles, $\alpha_{r f}$, for the MODERN pulses across the coil: $\alpha_{r f} \in \leq 90^{\circ}$. This flip angle distribution primarily affects the depth of dipolar oscillations (Ref. ${ }^{1}$, Figs. 4a and 6b). Such behavior is different from traditional $Y^{-}$ encoded RN pulses ${ }^{3,4}$, in which the scaling factor is affected, meaning the apparent dipolar oscillation frequency changes. (Ref. ${ }^{1}$, Figs. $4 a$ and $6 a$ ).

Figure S1A demonstrates the amplitude of the first minima of the MODER5 curve (the colormap) as functions of the pulse flip angle (axis X) and the input dipolar coupling values (axis Y). Under ideal conditions (zero offset and $90^{\circ}$ the flip angle), the amplitude of the first minima reaches -0.41 (dark blue). With increasing flip angle deviations from $90^{\circ}$, the performance of 
MODERN pulses deteriorates, and the amplitude of the first minima is shifted to positive values (red).

The sensitivity to the flip angle deviation is increased with decreasing dipolar coupling constant. For example, Figure S1B shows the MODER5 curves with $90^{\circ}$ flip angle (solid lines) and $85^{\circ}$ flip angle (dashed lines) with strong (23 kHz, black lines) and weak (2.3 kHz, red lines) dipolar coupling values. With a $23 \mathrm{kHz}$ dipolar coupling, MODER5 curves reach negative values for both cases. With a $2.3 \mathrm{kHz}$ coupling, MODERN curves coincide up to $\sim 0.1 \mathrm{~ms}$. From that time the solid red curve continues to dephase (eventually reaching -0.41), whereas the dashed red line returns toward 1.

The stability of the fitting procedure with respect to RF power can be assessed by first simulating MODER5 signal under different flip angle values and input dipolar coupling values ( $D_{\text {Is,intput }}$ ), and then fitting the simulated data (assuming perfect a flip angle) to obtain an output dipolar coupling value ( $\left.\mathrm{D}_{\text {IS,output }}\right)$. Again, the ideal MODER5 signal is obtained for a $90^{\circ}$ flip angel $\left(\alpha_{\mathrm{rf}}=90^{\circ}\right)$, The colormap in Figure S1C demonstrates the ratio between these two values, $\mathrm{D}_{\mathrm{IS}, \text { output }} /$ $D_{\text {IS,intput. }}$ In the ideal case this ratio is 1 (cyan). Above $D_{\text {IS, intput }}$ of $11 \mathrm{kHz}$, the output dipolar coupling is larger, while below the fit deviates towards smaller values. The deviations are symmetric with respect to the optimal value of $\alpha_{\mathrm{rf}}$ as was observed for the dependence of the MODER5 amplitude (Fig S1A). As expected from Fig S1C, stronger dipole couplings result in a broader region of

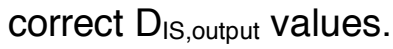
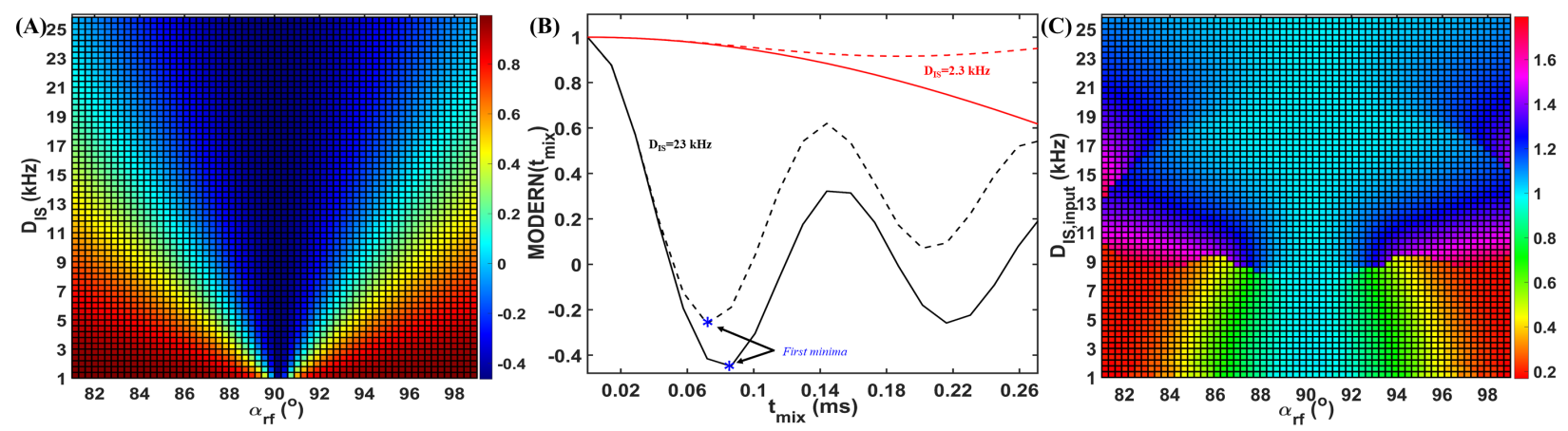

Figure S1. Simulated dependence of MODER5 pulses on flip angle deviations. (A) The value of the first minimum (marked with a star in B) as a function of a flip angle ( $a_{r}$, axis $x$ ) and the heteronuclear dipolar coupling values ( $D_{1 s}$, axis y). The MAS rate is $55.555 \mathrm{kHz}$. (B) The simulated MODER5 curves with strong ( $23.3 \mathrm{kHz}$, black lines) and weak

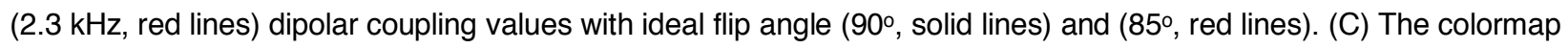

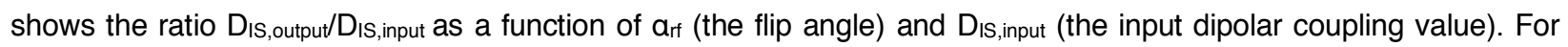
each pixel the simulated MODER5 ( $\left.a_{r f}, D_{i s, \text { input }}\right)$ was simulated and compared with MODER5 ( $a_{r}=90^{\circ}, D_{l s}$, output $)$ using

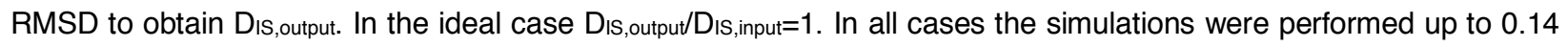
ms 
Although such sensitivity to the flip angle deviation (and rf-field inhomogeneity) impedes the measurements of the weak dipolar couplings, it can be taken into account into simulations in order to accurately measure stronger couplings, as shown below.

To confirm the reliability and the preciseness of the MODERN experiments for dipolar and torsion angle determinations, we investigated two samples: a-PET SH3 and fully protonated $\mathrm{SH} 3$ samples. a-PET SH3 sample was investigated twice: with short and long cross polarization (CP) transfer times from protons to carbons. For distinction, the datasets with a short and long $\mathrm{CP}$ are mentioned here as: a-PET SH3 (short CP) and a-PET SH3 (long CP).

To obtain the torsion angle values, the $\mathrm{HC}$ and $\mathrm{NH}$ dipolar coupling values first need to be determined. For the determination of the dipolar coupling values, MODERN pulses are applied on the proton channel while the coherence is on carbon spins ( $\mathrm{HC}$ coupling), or nitrogen spins ( $\mathrm{HN}$ coupling).

For each sample, the first step in the experimental protocol was a careful optimization of the MODER5 power level by maximizing the signal dephasing at short times. For example, at $\sim 0.07 \mathrm{~ms}$ a series of 1D spectra can be recorded as function of the rf-field power. The optimal rffield power is determinate when the MODERN signal has the lowest value. Figure S2A demonstrates the experimental HC MODER5 curve at $72 \mu$ s (expected first MODERN minimum for a $23 \mathrm{kHz}$ dipolar coupling) as a function of the proton rf-field power in Watts. The applied optimal rf-field powers on $\mathrm{HC}$ and $\mathrm{NH}$ dipolar interactions were optimized separately. In our case, the applied proton rf-field powers to recouple $\mathrm{HC}$ and dipolar interactions had the same values of $\sim 133 \mathrm{kHz}$ (with respect to the $90^{\circ}$ pulse calibration) for the $599 \mathrm{MHz}$ magnet; $128.5 \mathrm{kHz}$ and 129 $\mathrm{kHz}$ for the $800 \mathrm{MHz}$ magnet and $218 \mathrm{kHz}$ and $222 \mathrm{kHz}$ for the $950 \mathrm{MHz}$ magnet.

In addition to the influence of the rf-field inhomogeneity, we also found that the values of the proton to carbon CP times affected the performance of MODERN. Figure S3B demonstrates 1D HC MODER5 signal with a $72 \mu$ s MODER5 mixing time as a function of the CP time. Short mixing of $0.3 \mathrm{~ms}$ resulted in better performance than longer times.

After optimization of CP and rf-field powers, these same settings are used for dipolar coupling and torsion angle determination using MODERN.

For obtaining the dipolar coupling and torsion angle values, the experimental signals are compared with simulation in MATLAB (details in 'The MATLAB Code' section). Since the signal evolution is sensitive to the precise $B_{1}$ rf-field, the influence of the rf-field inhomogeneity in the 
probes should be simulated. The profile of rf-field inhomogeneity in MAS probes was investigated before $^{5-7}$. In these works, it was found that balanced probes result in symmetric profiles that are describe well by a Gaussian function. Since MODERN pulses have a symmetry around the optimal value (Fig S1), we used a half Gaussian function in simulations.

We found that reliable fits required consideration of rf inhomogeneity, which was modeled as a Gaussian flip angle distribution between 1 and a minimal value (maximum deviation, $\Delta \alpha_{r f, \max }$ :

$$
\Delta \alpha_{r f, \max }=1-\frac{\alpha_{r f, \min }}{90^{\circ}} \quad \text { Eqn. (S2) }
$$

To confirm the Gaussian profile of the rf-field inhomogeneity in the probe we recorded a series of $1 \mathrm{D} \mathrm{HCaNH}$ experiments as a function of the length of the first proton pulse from 1.8 to $184.2 \mu$ s with a $1.6 \mu$ s step. Figure S2C shows the FFT of such signal (black stars), the Gaussian function (red line) and half of the flip angle distribution function, which is used in simulations (cyan dashed line). All experimental and simulated FFT spectra coincide very well and symmetry around maximal value is observed.
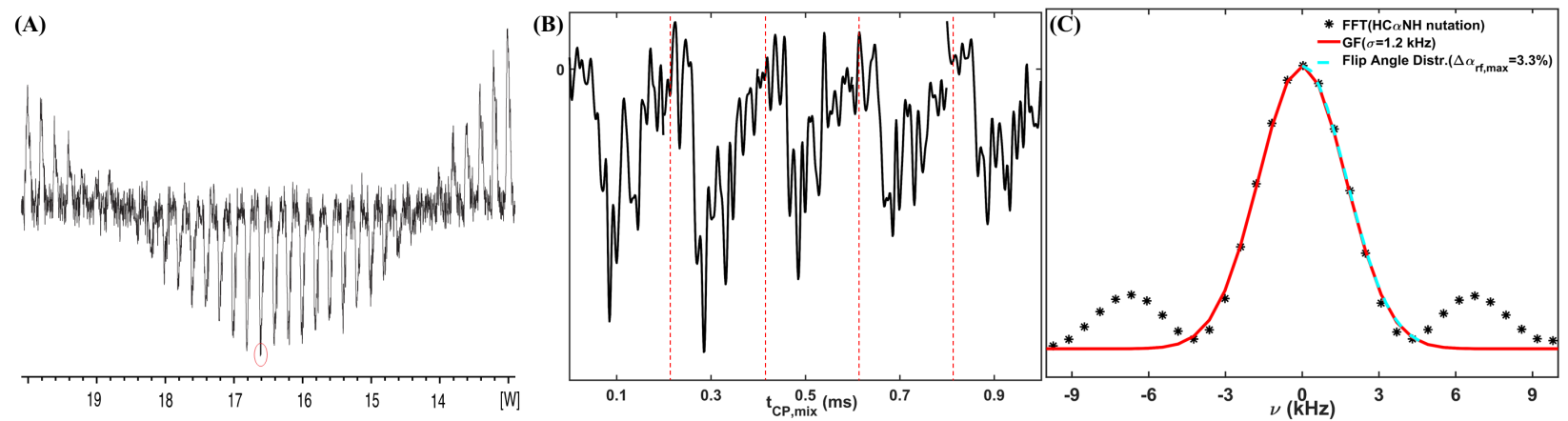

Figure S2. MODER5 optimization. (A) A series of 1D a-PET SH3 spectra obtained as a function of proton rf power (HC MODER5 pulses) measured in Watts. The recoupling time was set to $72 \mu \mathrm{s}$, which is the expected negative maximum for a $23 \mathrm{kHz}$ dipolar coupling. The red circled point was taken as the optimal power level for $137 \mathrm{kHz}$ proton recoupling. (B) MODER5 HC a-PET SH3 signal (with $72 \mu \mathrm{s}$ of MODER5 and optimized power level) as a function of the CP time (the CP transfer from proton to carbon before MODER5). (C) Fast Fourier Transform (FFT) of 1D (HCAN)H signal as a function of the length of first proton pulse (black stars) and comparison with a Gaussian function (red solid line) and a flip angle distribution function (cyan dashed line). The sample was the influenza A M2 protein. For FFT, MATLAB software was used. The experiments were performed at $55.555 \mathrm{kHz}$ MAS.

For a specific dipolar coupling value we simulate 7-14 curves with different values of the flip angle deviations $\Delta \alpha_{r f, i} \in\left[0: \Delta \alpha_{r f, \max }\right]$. In addition, we fit an effective exponential relaxation time, $T_{2, e f f}$. The total simulated MODERN signal is defined as follows:

$$
\bar{S}_{s i m}\left(v_{D} ; t_{m i x}\right)=\sum \bar{P}\left(\Delta \alpha_{r f, i}\right) S_{s i m}\left(v_{D} ; \Delta \alpha_{r f, i} ; t_{m i x}\right) e^{\left\{-t_{m i x} / T_{2, e f f}\right\}}, \quad \text { Eqn. (S3) }
$$


where $T_{2, e f f}$ is an effective relaxation time and $\bar{P}\left(\Delta \alpha_{r f, i}\right)$ is the normalized Gaussian weight factor for $\Delta \alpha_{r f, i}$ value.

To obtain these two values $\left(T_{2, e f f}, \Delta \alpha_{r f, \max }\right)$ several MODERN dipolar curves should be prepared as an input file (described in 'The MATLAB Code' section). The best MODERN curves for determination of these parameters are the curves where the oscillations with the largest amplitudes are observed and the longest MODERN mixing time is applied. For example, in our experiments the MODERN mixing time to recouple $\mathrm{NH}$ dipolar interactions was two times longer than for recoupling $\mathrm{HC}$ dipolar interactions. Therefore, we chose at least three NH MODER5 curves for each sample. Table S1 summarizes the obtained parameters. For a-PET SH3 samples (short and long CP) $T_{2, \text { eff }}$ has a smaller influence than for fully protonated SH3. The a-PET SH3 (short CP) dataset had the smallest apparent value of $\Delta \alpha_{r f, \max }$, suggesting that the CP time may influence the special distribution of the detected signal e.g. the edges versus the middle of the coil.

Table S1 The obtained $\Delta \alpha_{r f, \max }$ and $T_{2, e f f}$ from comparison of four experimental NH MODERN curves (for each sample) with simulated curves. The obtained values are average values obtained from four residues for a-PET SH3 (short CP) dataset and three residues for the other two samples.

\begin{tabular}{|c|l|l|l|}
\hline sample & $\alpha$-PET SH3 (short CP) & $\alpha$-PET SH3 (long CP) & SH3 (long CP) \\
\hline \multicolumn{1}{|c|}{$\Delta \alpha_{r f, \max }$} & 0.028 & $0.093 \pm 0.037$ & $0.078 \pm 0.01$ \\
\hline$T_{2, \text { eff }}(\mathrm{ms})$ & $0.648 \pm 0.23$ & $0.72 \pm 0.27$ & $0.257 \pm 0.014$ \\
\hline
\end{tabular}

Figure S3 shows the experimental (V9:NH, red stars) and simulated MODERN curves (lines). The dashed lines show an ideal experiment, with no experimental imperfection, such that $\Delta \alpha_{r f, i}=0$ and $T_{2, e f f} \gg t_{\text {exp }}$. The data clearly do not fit this case, but fit well when non-ideal values of $\Delta \alpha_{r f, \max }$ and $T_{2, e f f}$ are considered (solid line).
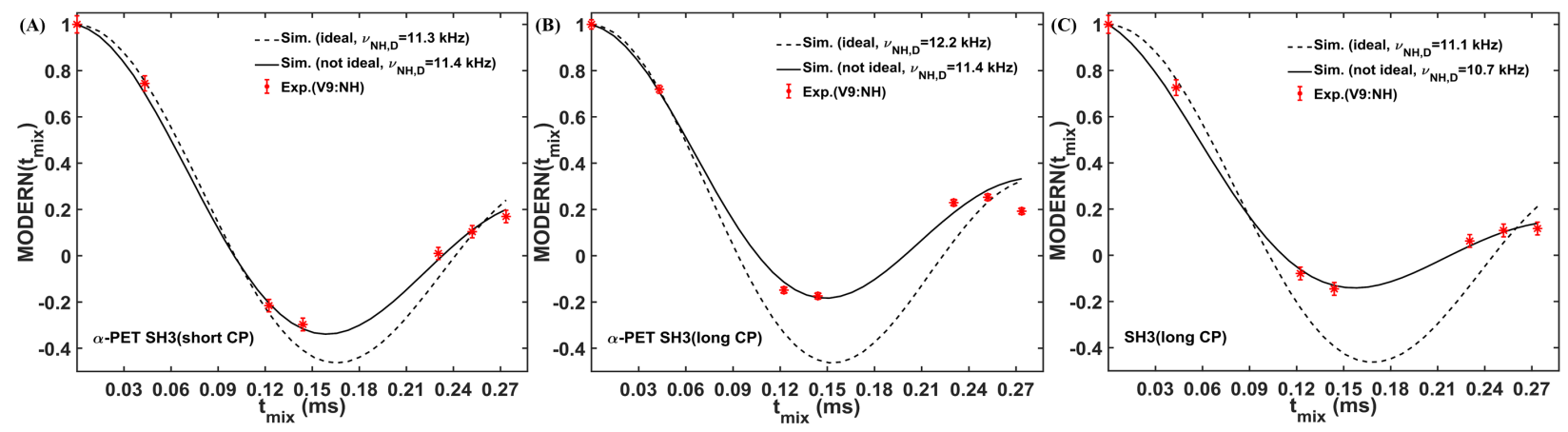

Figure S3. Experimental MODERN curves (V9: $\mathrm{NH}$, red stars) and simulated curves with ideal (dashed lines) and nonideal (solid lines) fit parameters. (A) a-PET SH3 with short CP. For non-ideal MODERN curves, $\Delta \alpha_{r f, \max }=0.028$ and 
$T_{2, e f f}=0.648 \mathrm{~ms}$. (B) a-PET SH3 with long CP mixing time. For non-ideal MODERN curves, $\Delta \alpha_{r f, \max }=0.093$ and $T_{2, e f f}=0.721 \mathrm{~ms}$. (C) SH3 with long CP mixing time. For non-ideal MODERN curves: $\Delta \alpha_{r f, \max }=0.078$ and $T_{2, e f f}=$ $0.257 \mathrm{~ms}$. Measurements are performed a $55 \mathrm{kHz}$ MAS. The solid lines represent the best simulated MODER 5 curves with the smallest RMSD values.

Using the obtained $\Delta \alpha_{r f, \max }$ and $\mathrm{T}_{2, \text { eff }}$ from Table $\mathrm{S} 1$, we obtained $\mathrm{HC}$ and $\mathrm{NH}$ dipolar coupling values for a-PET SH3 (short CP and long CP) and SH3 (long CP). Figures S4-S5 show the experimental and simulated MODER5 curves for a-PET SH3 (short CP) and SH3 (long CP). 

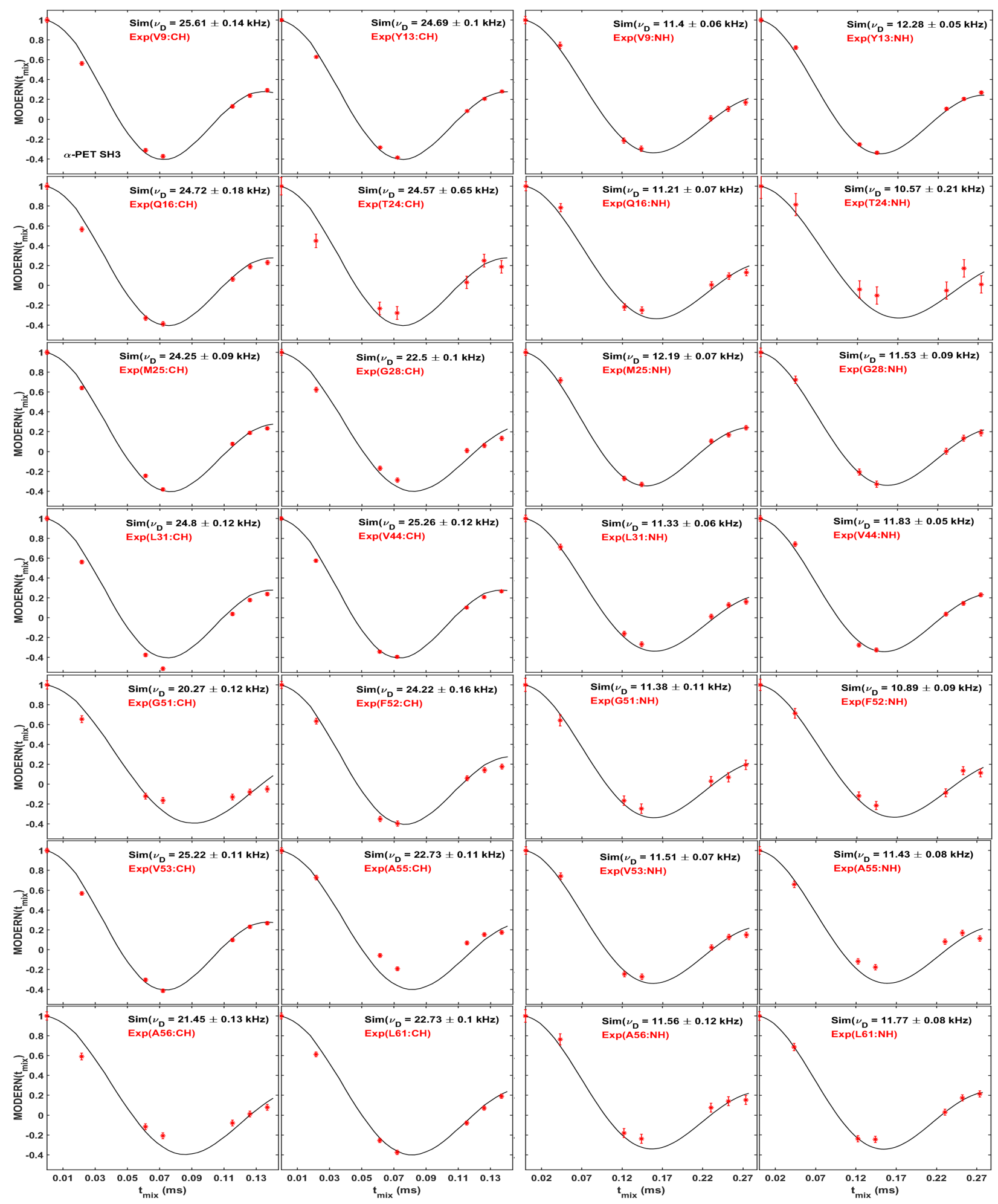

Figure S4. Experimental a-PET SH3 (short CP) and simulated MODER5 curves for HC (left two columns) and NH (right two columns) dipolar coupling values. The simulated MODER5 curves were obtained with the $\Delta \alpha_{r f, \max }$ and $T_{2, e f f}$ values of Table S1). Eqn. S3 represents the simulated signal. The fitting errors were obtained by generating 100 Monte Carlo curves, assuming a Gaussian noise distribution ( 1.5б). 

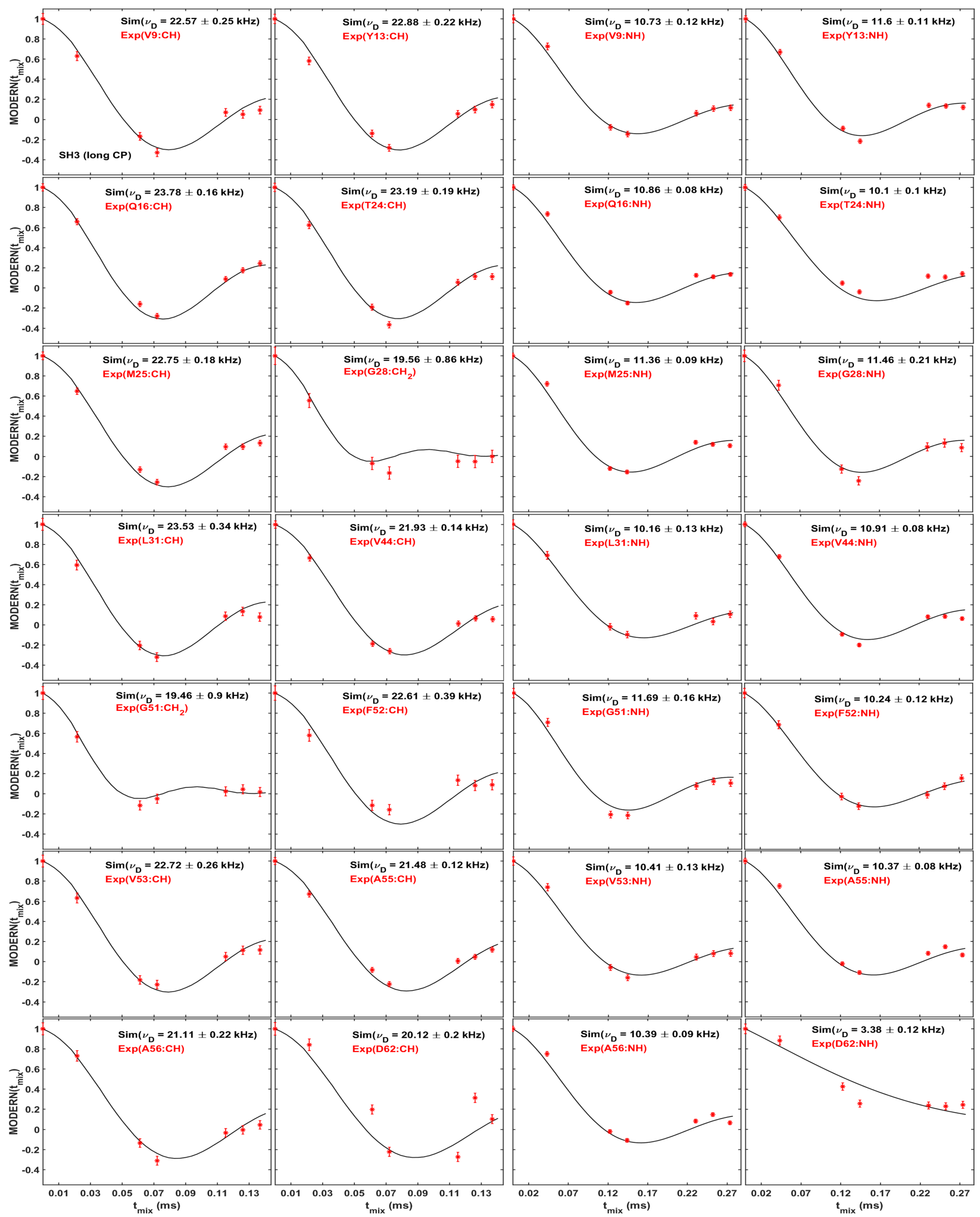

Figure S5. Experimental SH3 (long CP) and simulated MODER5 curves for HC (left two columns) and $\mathrm{NH}$ (right two columns) dipolar coupling values. The simulated MODER5 curves were obtained with the $\Delta \alpha_{r f, \max }$ and $T_{2, \text { eff }}$ values 
of Table S1). Eqn. S3 represents the simulated signal. The fitting errors were obtained by generating 100 Monte Carlo curves, assuming a Gaussian noise distribution $(\sim 1.5 \sigma)$.

For processing the a-PET SH3 (long CP) MODERN data, we had to add an additional parameter, $\mathrm{p}$, which defined the population of protonated $C_{a}$ signal (Figure S6A). The particular a-PET sample used had incomplete incorporation of alpha protons (due to incomplete production of keto acids in the enzymatic digestion step) The deuterated alpha carbons, $D_{\alpha} C_{\alpha}$, are increasingly polarized at longer CP times, e.g. from nearby protons such as the backbone amide proton. The signal from this part of the sample is then transferred on through CP to nitrogen and then detected at the amide proton. However, there can be no $\mathrm{CH}$ MODERN oscillation for this part of the sample. The dipolar interaction between $C_{a}$ and remote protons is $\sim 8$ times weaker in comparison to directly bonded protons. At short CP mixing times, only directly bonded $\mathrm{HC}$ groups are excited. However, at long mixing times, the total CP signal is the sum of the strong (directly bonded protons) and weak (remote protons) $\mathrm{CH}$ groups. Note that $\mathrm{p}$ is not a labelling population, but also depends on the local geometry of nearby protons, which explains the different $p$ values fit to different residues of the same type (Figure S8).

For a-PET SH3 (long CP) Eqn. (3) is then rewritten as follow:

$$
\begin{gathered}
\bar{S}_{s i m}\left(v_{D} ; t_{m i x}\right)= \\
\sum \bar{P}\left(\Delta \alpha_{r f, i}\right)\left[p \cdot S_{s i m}\left(v_{D} ; \Delta \alpha_{r f, i} ; t_{m i x}\right)+(1-p) \cdot S_{s i m}\left(v_{D} / 8 ; \Delta \alpha_{r f, i} ; t_{m i x}\right)\right] e^{\left\{-t_{m i x} / T_{2, e f f}\right\}} \text {. Eqn. (S4 }
\end{gathered}
$$

Figure S6B shows the experimental MODER5 V9 HC curve (red stars) and simulations (solid lines), assuming full (black) and partial (red) protonation of the backbone $C_{\alpha}$ carbons.

(A)

Fully protonated backbone:

$$
\mathbf{p}=\mathbf{1}
$$

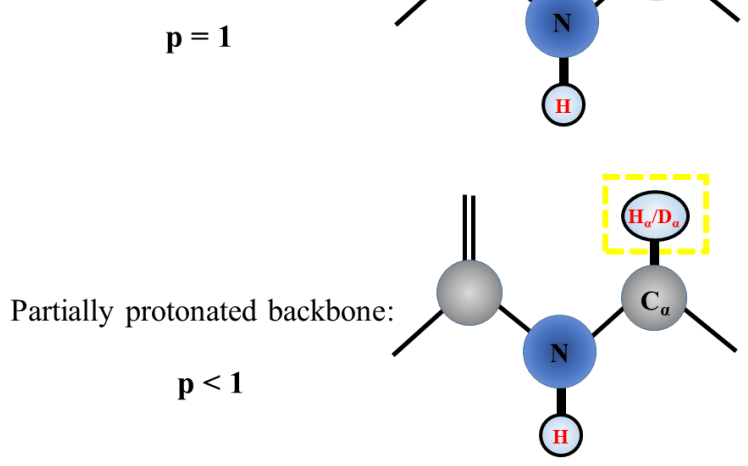

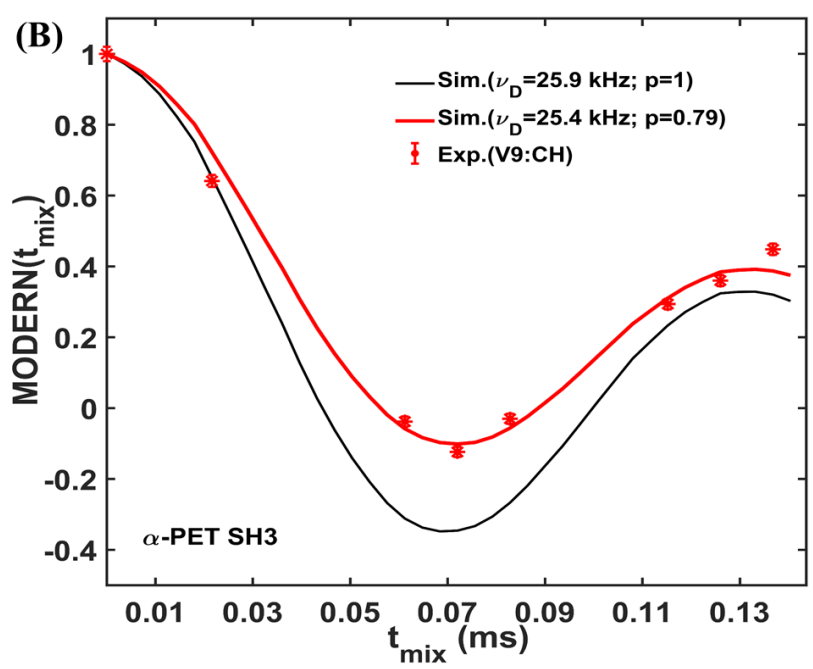

Figure S6. Partial protonation of backbone $C_{\alpha}$ carbons in a-PET SH3 sample. (A) The schematic presentation of two cases: all proteins with fully protonated backbones (upper, $p=1)$ and some proteins $(1-p, p<1)$ have deuterated 
backbone (lower). (B) Experimental MODER5 V9 HC curve (red stars) and comparison of the simulated MODER5 curves (solid lines) with fully protonated backbone assumption ( $\mathrm{p}=1$, black line) and partially protonated backbone $C_{a}$ carbon assumption ( $\mathrm{p}=0.79$, red line).

Figures S7 shows the experimental and simulated MODER5 curves for a-PET SH3 (long $\mathrm{CP})$. 

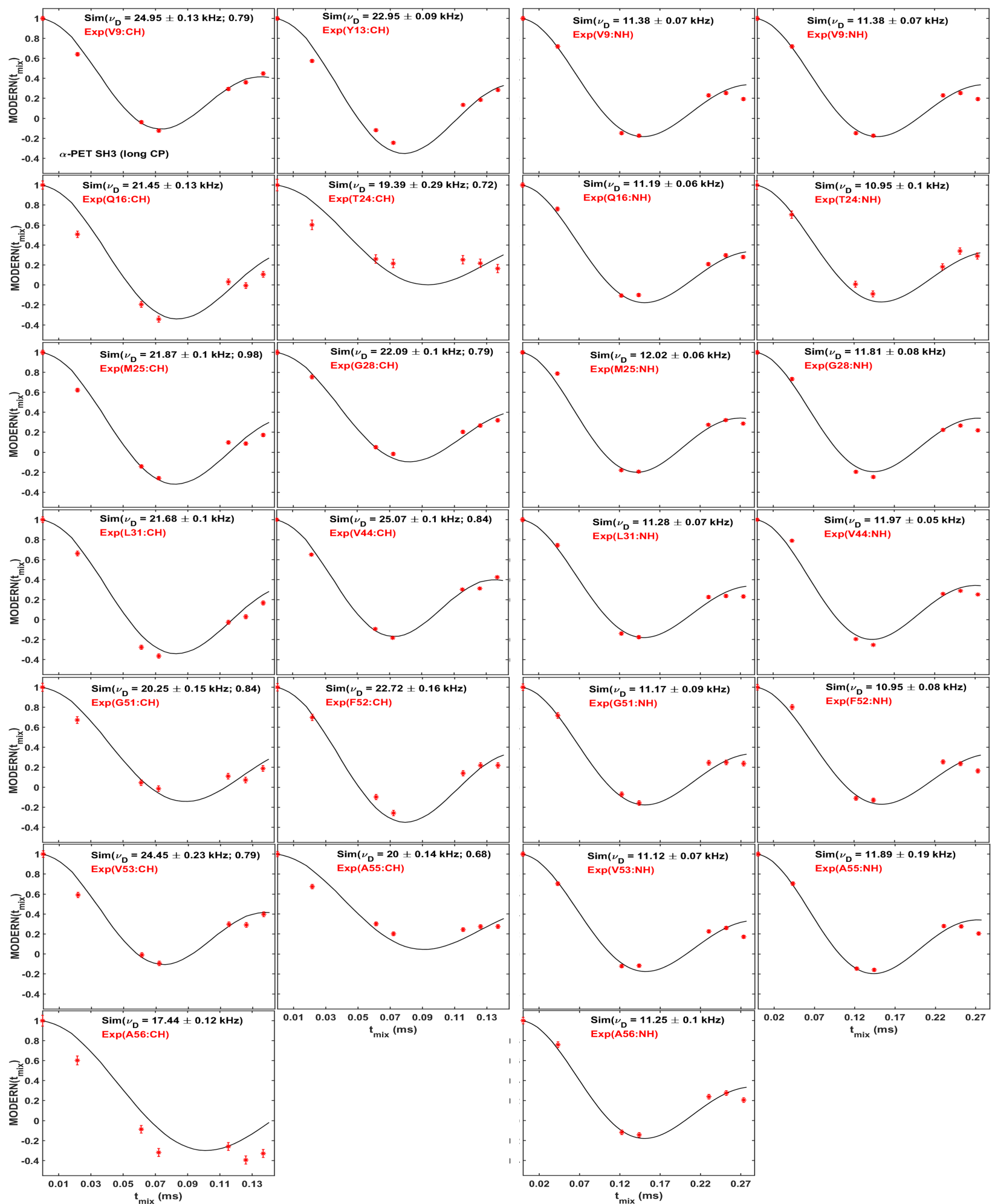

Figure S7. Experimental a-PET SH3 (long CP) and simulated MODER5 curves for HC (left two columns) and NH (right two columns) dipolar coupling values. The simulated MODER5 curves were obtained with $\Delta \alpha_{r f, i}$ and $T_{2, e f f}$ (Table S1). Eqn. S4 represents the simulated signal. The fitting errors were obtained by generating 100 Monte Carlo curves, assuming the Gaussian noise distribution $(\sim 1.5 \sigma)$. 
Figure $\mathrm{S} 8$ shows the population of the protonated backbone $C_{a}$ carbons for different residues. For some residues, $C_{a}$ are fully protonated (Q16 and L31), whereas for others an apparent $35 \%$ of the signal comes from deuterated alpha carbon (T24).

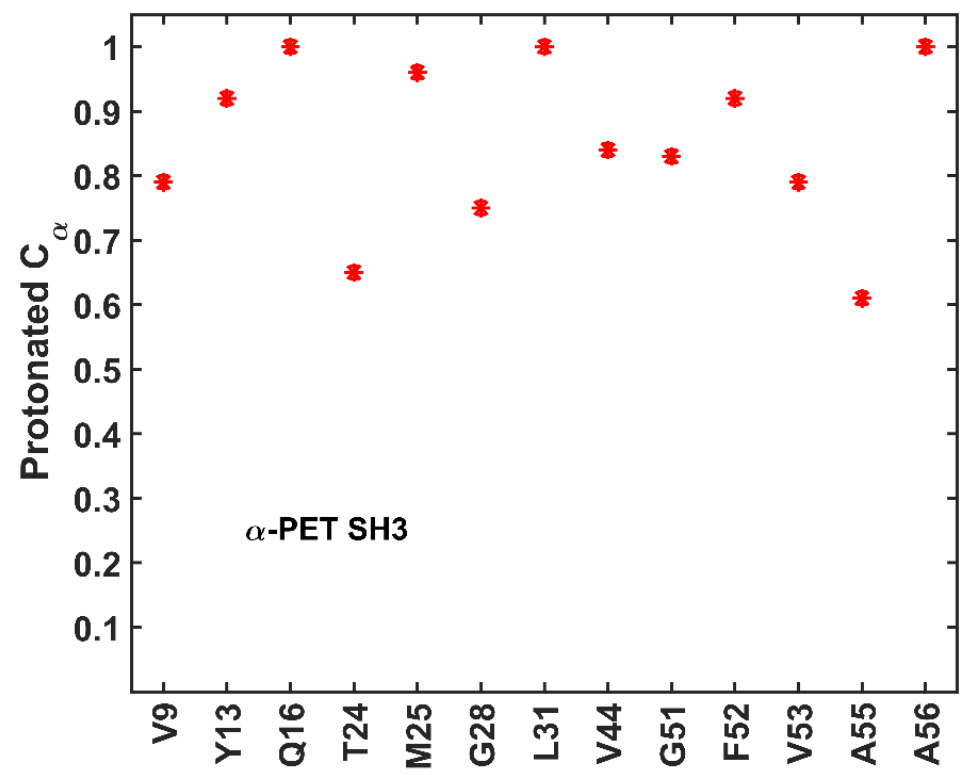

Figure S8. The fraction of signal arising from protonated backbone $C_{a}$ carbons ( $p$, axis $\mathrm{Y}$ ) for different residues (axis $\mathrm{X}$ ) for a-PET $\mathrm{SH}_{3}$ sample. The population, p, was obtained from the comparison of experimental $\mathrm{CH}$ and simulated MODER5 curves.

Figure S9 summarizes the obtained $\mathrm{HC}(\mathrm{A})$ and $\mathrm{NH}$ (B) dipolar coupling values for all three samples. Additionally we compare NH dipolar coupling values, which were obtained by off-MAS method (cyan squares), of Xue et al (Ref. $\left.{ }^{8}\right)$.
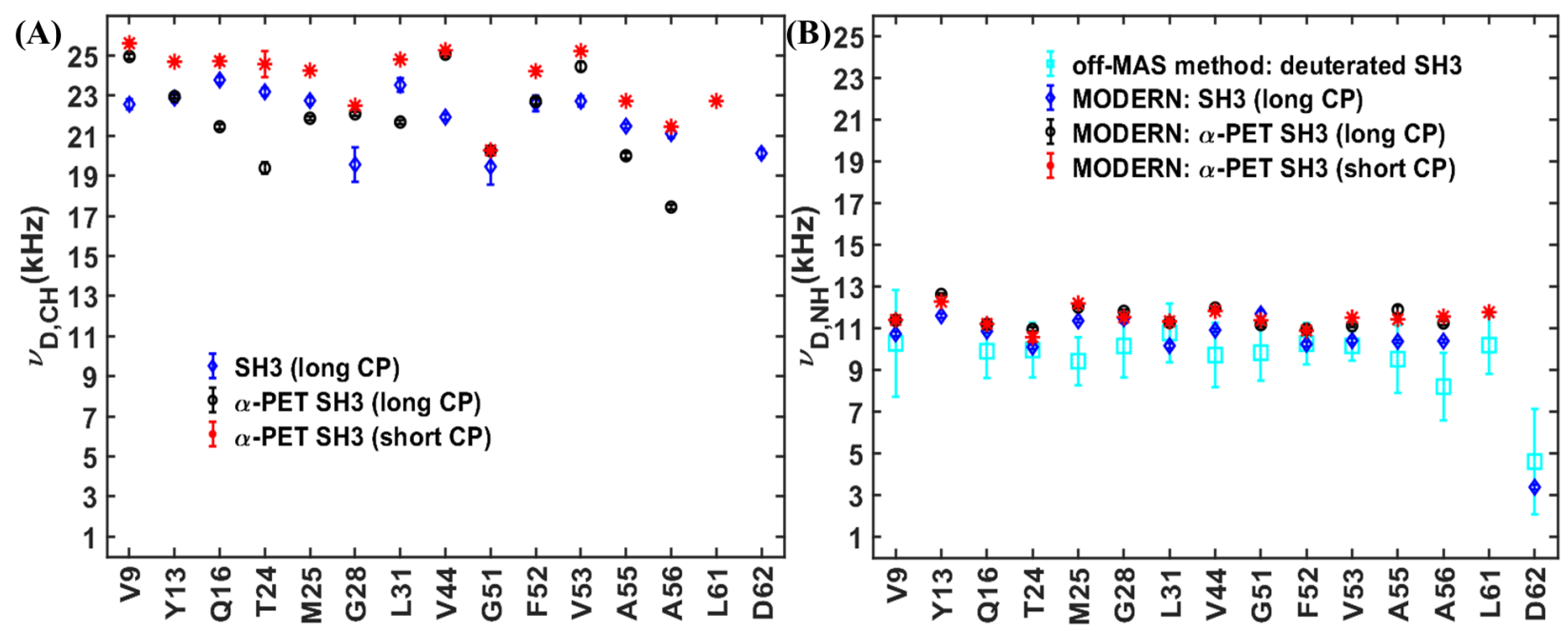
Figure S9. The obtained $\mathrm{HC}(\mathrm{A})$ and $\mathrm{NH}(\mathrm{B})$ dipolar coupling values for different samples: $\mathrm{SH} 3$ (long $\mathrm{CP}$ ) - blue diamonds; a-PET SH3 (long CP) - black circles and a-PET SH3 (short CP) - red stars. In (B) cyan squares represent the NH dipolar coupling values, which were obtained by off-MAS method from deuterated SH3.

\section{Torsion Angle Determination}

The sequential application of MODERN pulses to recouple both $\mathrm{HC}$ and $\mathrm{NH}$ dipolar interactions allows measurement of the projection angle between these interactions, $\phi_{p r o j}$. The projection angle can be visualized by translation of the $\mathrm{HC}$ bond to the $\mathrm{NH}$ bond such that $\mathrm{C}$ and $\mathrm{N}$ atoms coincide.

Figure S10 demonstrates the simulated dependence of MODER5-MODER5 curves on the projection angle for $\mathrm{HC} / \mathrm{NH}$ (Figure $\mathrm{S} 11 \mathrm{~A}$ ) and $\mathrm{H}_{2} \mathrm{C} / \mathrm{NH}$ (Figure $\mathrm{S} 10 \mathrm{~B}$ ) groups. The dependence on the projection angle is shown between $0^{\circ}$ and $90^{\circ}$, since the curves with $\phi_{\text {proj }}$ and $180^{\circ}-\phi_{\text {proj }}$ have almost identical behaviors at short mixing times. Comparing the simulated curves in Figure $\mathrm{S} 10 \mathrm{~A}$ with the curves in Figure $\mathrm{S} 10 \mathrm{~B}$, it is clear that $\mathrm{HC} / \mathrm{NH}$ curves are more sensitive to $\phi_{\text {proj }}$ than $\mathrm{H}_{2} \mathrm{C} / \mathrm{NH}$ curves, in particular below $0.05 \mathrm{~ms}$. It shows the advantage of measuring the torsion angles with the deuterated a-PET SH3 sample, in which Ca of GLY residues (G28 and G51) contain only one proton and the second is replaced with deuterium.

Figure $\mathrm{S} 11 \mathrm{C}$ shows simulated $\mathrm{HC} / \mathrm{NH}$ curves, taking into account the experimental imperfections for a-PET SH3 with long CP $\left(\Delta \alpha_{r f, \max }=0.1\right.$ and $0.55 \mathrm{~ms}$ of $\left.T_{2, \text { eff }}\right)$. This Figure shows that the angle can still be precisely determined in the presence of RF inhomogeneity.
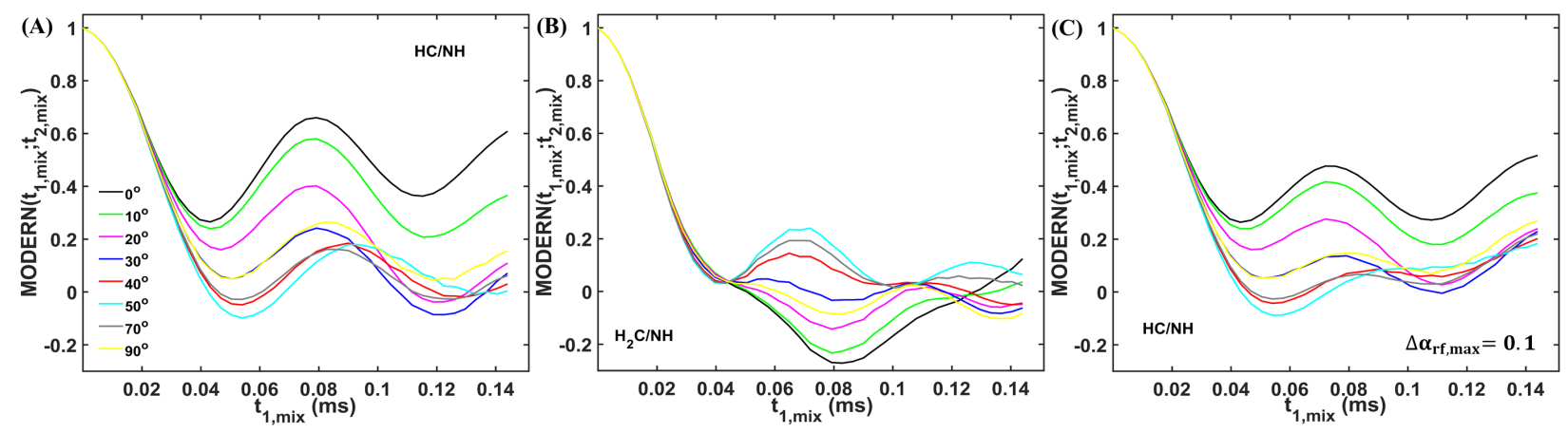

Figure S10. Simulated MODER5-MODER5 recoupling curves with different projection angle geometries $\left(\phi_{\text {proj }}\right)$. For $\mathrm{HC}$ and $\mathrm{NH}$ groups, $\mathrm{D}_{\mathrm{HC}}=22 \mathrm{kHz}$ and $\mathrm{D}_{\mathrm{HN}}=11 \mathrm{kHz}$. The MAS rate is $55.555 \mathrm{kHz}$. The mixing time on $\mathrm{NH}$ dipolar pair ( $t_{2, \text { mix }}$ ) is 2 times longer than on $\mathrm{CH}$ dipolar pair $\left(\mathrm{t}_{1, \text { mix }}\right): \mathrm{t}_{2, \text { mix }}=2 \mathrm{t}_{1, \text { mix. }}(\mathrm{A})$ The projection angle between $\mathrm{HC} / \mathrm{NH}$ with ideal MODER5-MODER5 simulations $\left(\Delta \alpha_{r f, \max }=0\right)$. (B) The projection angle between $\mathrm{H}_{2} \mathrm{C}-\mathrm{NH}$. The $\mathrm{H}-\mathrm{C}-\mathrm{H}$ angle is $109^{\circ}$. (C) The projection angle between $\mathrm{HC} / \mathrm{NH}$ with $\Delta \alpha_{r f \text { max }}=0.1$.

However, the desired angle is a torsion angle, $\phi_{\mathrm{H}}$, which is produced by two planes defined by atoms $\mathrm{Ca}-\mathrm{N}-\mathrm{H}^{\mathrm{N}}$ and $\mathrm{Ha}-\mathrm{C}-\mathrm{N}$. The next Eqn. links the torsion and projection angles: 


$$
\cos \left(\phi_{\text {proj }}\right)=\sin \left(\theta_{H N C \alpha}\right) \sin \left(\theta^{\prime}{ }_{N C \alpha H \alpha}\right) \cos \phi_{H}+\cos \left(\theta_{H N C \alpha}\right) \cos \left(\theta^{\prime}{ }_{N C \alpha H \alpha}\right), \quad \text { Eqn. (S5) }
$$

where $\theta_{H N C \alpha}$ is the $\mathrm{H}^{\mathrm{N}}-\mathrm{N}$ - Ca angle; $\theta^{\prime}{ }_{N C \alpha H \alpha}=180^{\circ}-\theta_{N C \alpha H \alpha}$, where $\theta_{N C \alpha H \alpha}$ is the $\mathrm{NC}_{a} \mathrm{H}_{\mathrm{a}}$ angle.

Figure $S 11$ shows the conversion of the torsion angles, $\phi_{H}$, between [0॰:180 $]$ into the projection angle domain for specific values of $\theta_{H N C \alpha}$ and $\theta^{\prime}{ }_{N C \alpha H \alpha}$. Figure S11A considers two cases: $\theta_{H N C \alpha}=120^{\circ}$ whereas $\theta^{\prime}{ }_{N C \alpha H \alpha}$ is changed between $0^{\circ}$ and $90^{\circ}$ (blue area), and $\theta_{H N C \alpha}$ is changed between $90^{\circ}$ and $180^{\circ}$ whereas $\theta^{\prime}{ }_{N C \alpha H \alpha}=71^{\circ}$ (red area). For example, when $\theta_{H N C \alpha}=$ $120^{\circ}$ and $\theta^{\prime}{ }_{N C \alpha H \alpha}=0^{\circ}$, the projection angle is always $120^{\circ}$ for all $\phi_{H} \in\left[0^{\circ}: 180^{\circ}\right]$. When $\theta_{H N C \alpha}=$ $120^{\circ}$ and $\theta^{\prime}{ }_{N C \alpha H \alpha}=90^{\circ}$, the projection angles obtain values between [30 $150^{\circ}$ ]. In our case with $\theta_{H N C \alpha}=120^{\circ}$ and $\theta^{\prime}{ }_{N C \alpha H \alpha}=71^{\circ}$, the torsion angle range between $\left[0^{\circ}: 180^{\circ}\right]$ is converted into the projection angle range of [49:169].

Figure S11B shows the projection angle range, when $\theta^{\prime}{ }_{N C \alpha H \alpha}$ and $\theta_{H N C \alpha}$ are changed at the same time, while the difference $\theta_{H N C \alpha^{-}} \theta^{\prime}{ }_{N C \alpha H \alpha}=50^{\circ}$.
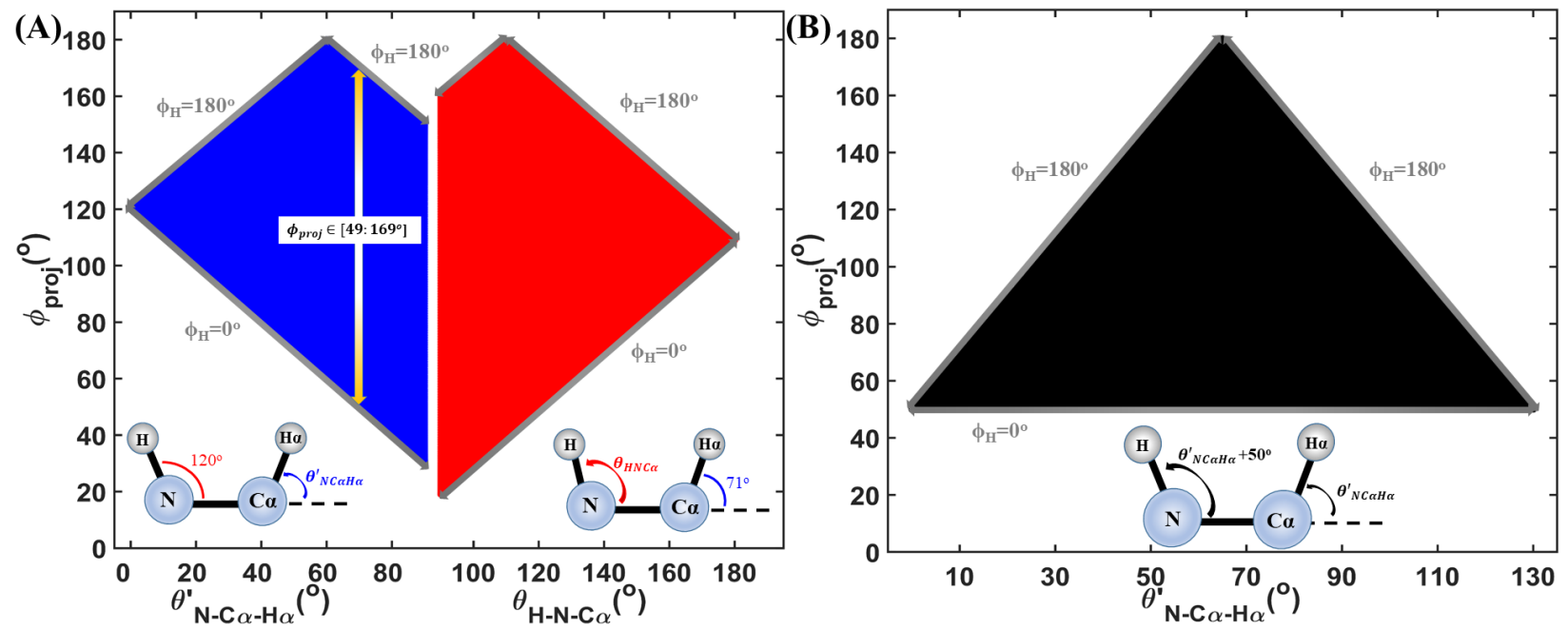

Figure S11. The dependence of the range of the projection angles, $\phi_{p r o j}$, on $\theta_{H N C \alpha}$ and $\theta^{\prime}{ }_{N C \alpha H \alpha}$ values for $\phi_{H} \in$ $\left[0^{\circ}: 180^{\circ}\right]$. (A) The projection angle range as a function of $\theta^{\prime}{ }_{N C \alpha H \alpha}$ (between $0^{\circ}$ and $90^{\circ}$ ) with constant $\theta_{H N C \alpha}=120^{\circ}$ (blue area) and as a function of $\theta_{H N C \alpha}$ (between $90^{\circ}$ and $180^{\circ}$ ) with $\theta^{\prime}{ }_{N C \alpha H \alpha}=71^{\circ}$. (B) The projection angle range as a function of $\theta^{\prime}{ }_{N C \alpha H \alpha}$ (between $0^{\circ}$ and $130^{\circ}$ ), with $\theta_{H N C \alpha}-\theta^{\prime}{ }_{N C \alpha H \alpha}=50^{\circ}$.

To obtain $\phi_{H}$ values we used the set of the $\mathrm{HC}$, NH dipolar coupling values, $\Delta \alpha_{r f, \max }$, and $T_{2, e f f}$ as the input parameters. For torsion angle determination, $\phi_{H}$ value was the only fit variable. Figures S12-S14 show the comparison of the experimental and simulated MODER5-MODER5 curves for all three samples. Figure 12 also shows a plot of Eqn. S5. 

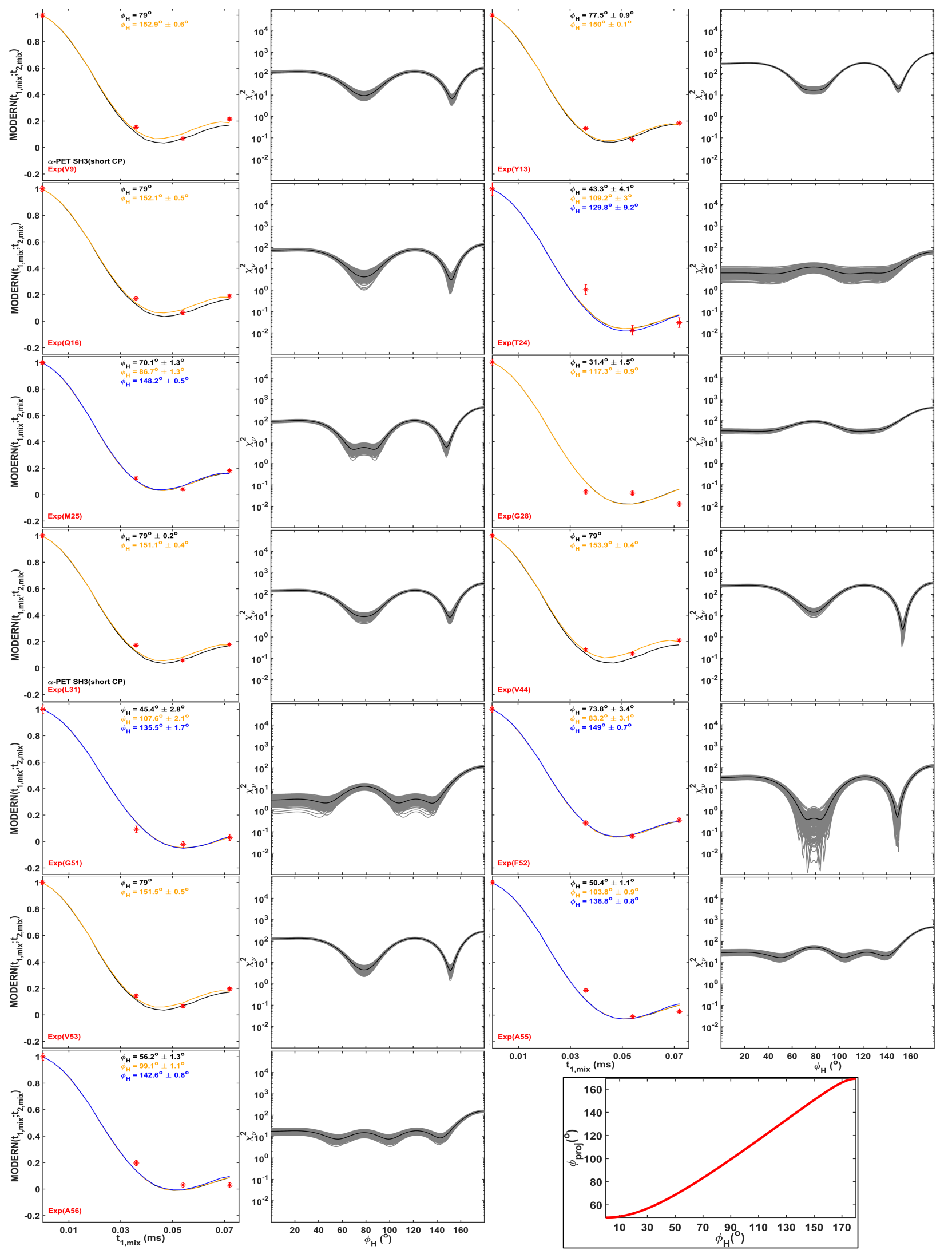

\begin{tabular}{|c|}
\hline $0^{-1}$ \\
$10^{-2}$ \\
$10^{4}$ \\
$10^{3}$ \\
\hline
\end{tabular}

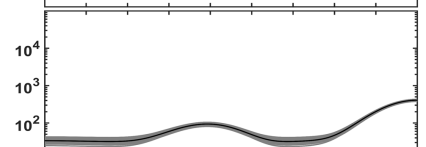

$\times \times 10^{1}$

$10^{0}$
$10^{-1}$

$10^{-1}$
$10^{-2}$
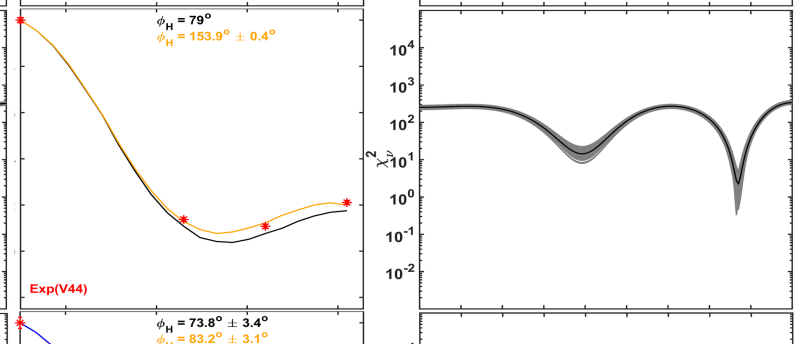

$10^{-1}$
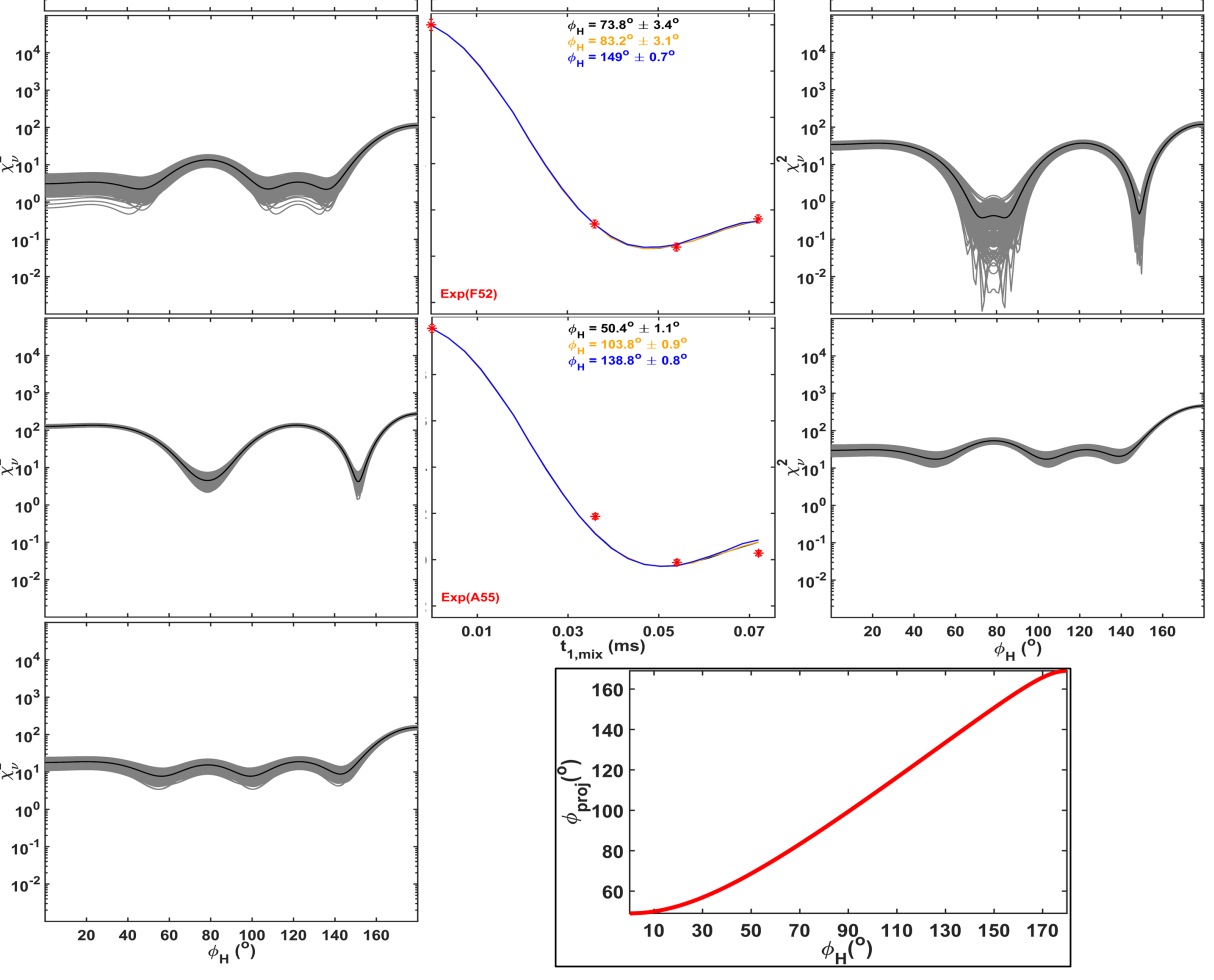
Figure S12. The first and third columns: Experimental a-PET SH3 (short CP) and simulated MODER5-MODER5 curves (solid lines). The second and fourth columns: $\chi_{v}^{2}$ (Eqn. S1) as a function of torsion angle, $\phi \mathrm{H}$. The simulated MODER5MODER5 curves were obtained with $\Delta \alpha_{r f, i}$ and $T_{2, e f f}$ values of Table $\mathrm{S} 1$ and measured dipolar coupling values. The fitting errors were obtained by generating 300 Monte Carlo curves (grey curves, the black curve is the average), assuming a Gaussian noise distribution $(\sim 1.5 \sigma)$ and considering the errors in the determination of dipolar coupling values. The Figure in the black frame shows the projection angle as a function of the torsion angle for $\theta^{\prime}{ }_{N C \alpha H \alpha}=71^{\circ}$ and $\theta_{H N C \alpha}=120^{\circ}$. 

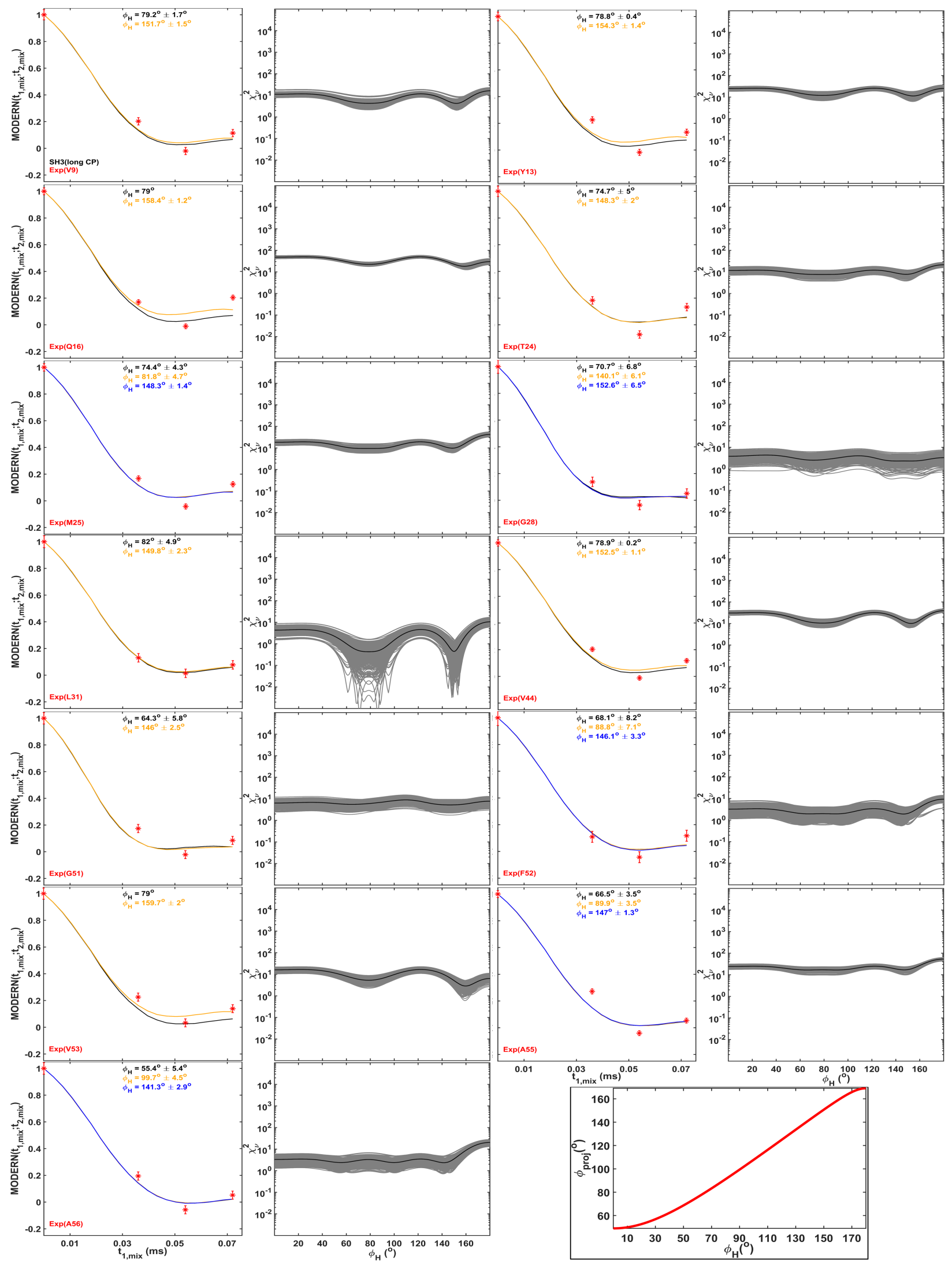
Figure S13. The first and third columns: Experimental SH3 (long CP) and simulated MODER5-MODER5 curves (solid lines). The second and fourth columns: $\chi_{v}^{2}$ (Eqn. S1) as a function of torsion angle, $\phi_{\mathrm{H}}$. The simulated MODER5MODER5 curves were obtained with $\Delta \alpha_{r f, i}$ and $T_{2, e f f}$ of Table S1 and measured dipolar coupling values. The fitting errors were obtained by generating 300 Monte Carlo curves (grey curves, the black curve is the average), assuming a Gaussian noise distribution $(\sim 1.5 \sigma)$ and considering the errors in the measured dipolar coupling values. The Figure in the black frame shows the projection angle as a function of the torsion angle for $\theta^{\prime}{ }_{N C \alpha H \alpha}=71^{\circ}$ and $\theta_{H N C \alpha}=120^{\circ}$. 

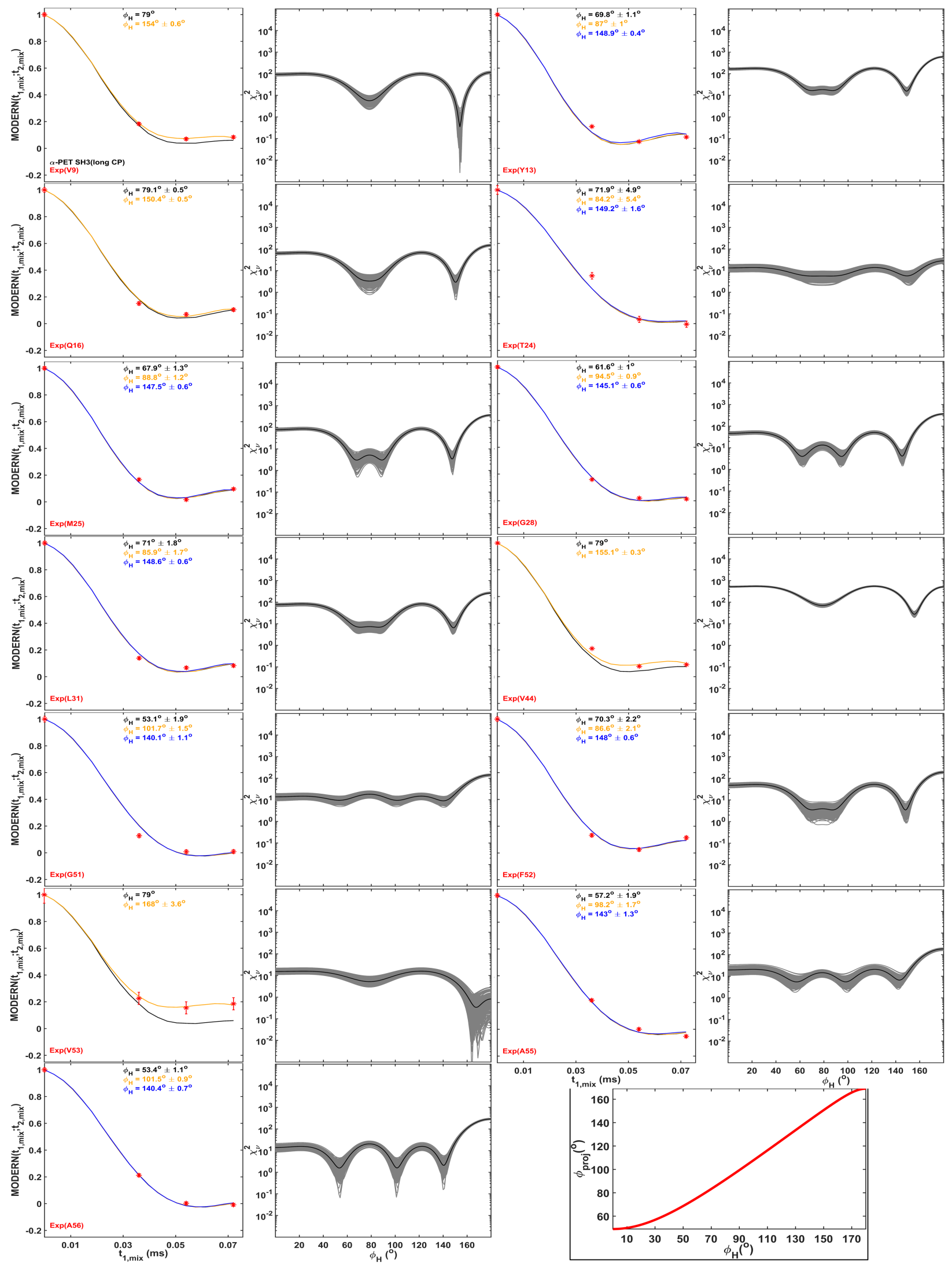
Figure S14. The first and third columns: Experimental a-PET SH3 (long CP) and simulated MODER5-MODER5 curves (solid lines). The second and fourth columns: $\chi_{v}^{2}$ (Eqn. S1) as a function of torsion angle, $\phi \mathrm{H}$. The simulated MODER5MODER5 curves were obtained with $\Delta \alpha_{r f, i}$ and $T_{2, e f f}$ values in Table $\mathrm{S} 1$ and measured dipolar coupling values. The fitting errors were obtained by generating 300 Monte Carlo curves (grey curves, the black curve is the average), assuming a Gaussian noise distribution $(\sim 1.5 \sigma)$ and considering the errors in the measured dipolar coupling values. The Figure in the black frame shows the projection angle as a function of the torsion angle for ${\theta^{\prime}}^{\prime}{ }_{N C \alpha H \alpha}=71^{\circ}$ and $\theta_{H N C \alpha}=120^{\circ}$.

Figure S15A summarizes the obtained torsion angles as a function of the x-ray crystallography-derived angles from the pdb file 2NUZ. Figure S15B compares the absolute differences between the obtained torsion angles from $\alpha$-PET SH3 using short CP and the x-ray crystallography-derived angles from pdb 2NUZ (red stars) and pdb 1SHG file (blue diamonds). These two structures have comparable unit cell parameters, but were reported independently from two different research groups. About a $5^{\circ} \mathrm{rms}$ deviation is observed between the two crystal structures. This can be compared with the $17.82^{\circ}$ and $17.31^{\circ} \mathrm{rms}$ deviation from the NMR data.
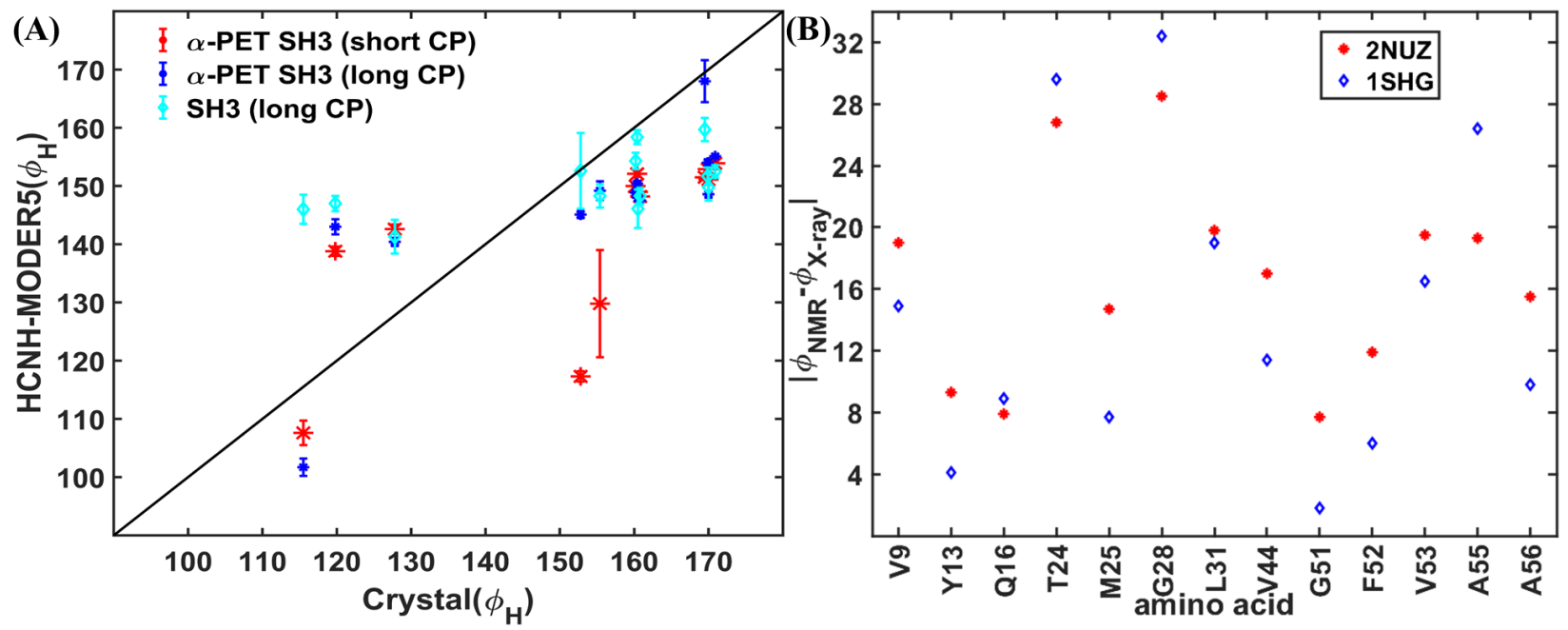

Figure S15. (A) Correlation of the torsion angles determined from X-ray crystallography (2NUZ file, axis $x$ ) and MAS NMR (axis y) methods. Red stars - a-PET SH3 (short CP) dataset; blue stars - a-PET SH3 (long CP) dataset and cyan diamonds - SH3 (long CP) dataset. (B) The absolute difference between the torsion angles from a-PET SH3 (short CP) sample and the torsion angles determined from 2NUZ file (red stars) and 1SHG file (blue diamonds).

Figure S16 demonstrates the experimental (red starts) and simulated (solid lines) MODER5 torsion angle curves of two residues W41 (A, C, E) and F47 (B, D, F) in three different M2 datasets and two samples, WT and S31N. M2 WT data was obtained with an $800 \mathrm{MHz}$ magnet and $55.555 \mathrm{kHz}$ MAS. The two other datasets (one for WT, one for S31N) were obtained with a $950 \mathrm{MHz}$ magnet and $90.909 \mathrm{kHz}$ MAS. The obtained torsion angles for different samples are in good agreement. The full experimental dataset for all resolved residues from the $950 \mathrm{MHz}$ magnet is shown in Figures S18-S22. 

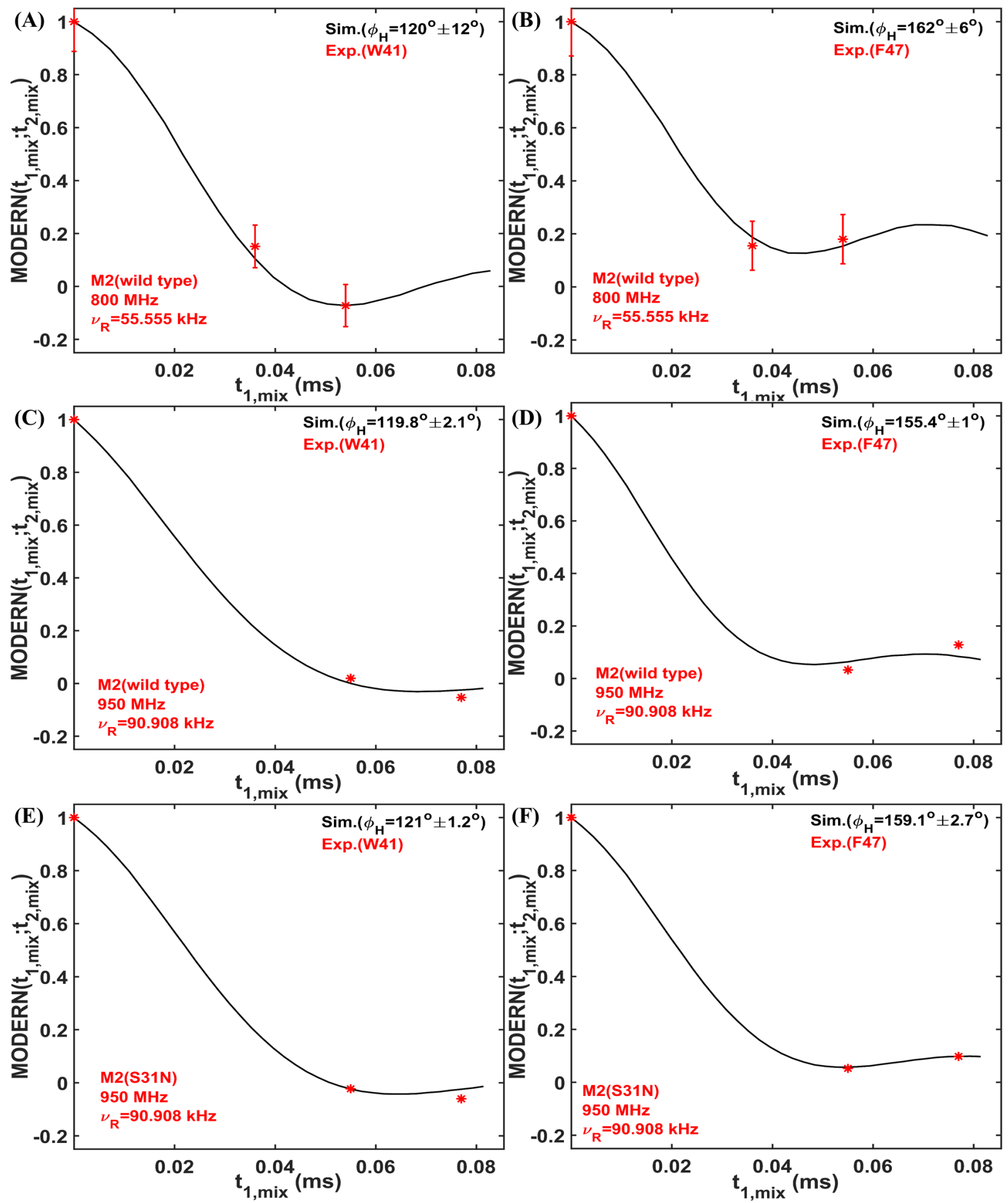

Figure S16. Best fit curves and torsion angle values for residues $\mathrm{W} 41(\mathrm{~A}, \mathrm{C}, \mathrm{E})$ and $\mathrm{F} 47(\mathrm{~B}, \mathrm{D}, \mathrm{F})$ obtained with $\mathrm{HC} \alpha \mathrm{NH}-$ MODER5 for M2 wild type (A, B, C, D) and M2 S31N (E, F). (A) and (B): Measurements were performed at an $800 \mathrm{MHz}$ spectrometer using a $1.3 \mathrm{~mm}$ probe with $55.555 \mathrm{kHz}$ MAS. (C)-(F): Measurements were performed at a $950 \mathrm{MHz}$ spectrometer using a $0.7 \mathrm{~mm}$ probe with $90.908 \mathrm{kHz}$ MAS. The data in Figures $(A)-(B)$ and $(C)-(F)$ were recorded as pseudo 4D and pseudo 3D experiments, respectively. 


\section{M2 data, $950 \mathrm{MHz}$ spectrometer}

This data was collected as pseudo 3D spectra at a $950 \mathrm{MHz}$ spectrometer with 90.908 $\mathrm{kHz}$ MAS. Firstly, we optimized MODER5 rf-field power, in the similar way as for $\mathrm{SH} 3$ samples keeping short CP values. Additionally, we found that with high MAS rate, the incline of ramp of $\mathrm{CP}$ has a significant influence on the MODERN performance, as measured by the depth of the oscillation. Figure S17 demonstrates 1D detected MODER5 NH signal at $154 \mu$ s mixing for S31N M2 for four different sets of ramps. The best performance is observed with $90 \% \rightarrow 100 \%$ for $\mathrm{HC}$ $\mathrm{CP}$ and $70 \% \rightarrow 100 \%$ for NH CP (red). The starting signal (without mixing) varied by $\sim 10$ percent among these CP conditions. The same dependence was found for M2 wild type. Table S2 summarizes the optimized parameters: $\Delta \alpha_{r f, \max }$ and $T_{2, e f f}$.

Table S1 The obtained $\Delta \alpha_{r f, \max }$ and $T_{2, \text { eff }}$ from comparison of three experimental NH MODERN curves (for each sample) with simulated curves.

\begin{tabular}{|c|c|c|}
\hline sample & M2 (S31N) & M2 (wild type) \\
\hline$\Delta \alpha_{r f, \max }$ & 0.028 & 0.028 \\
\hline$T_{2, \text { eff }}(\mathrm{ms})$ & $0.246 \pm 0.018$ & $0.194 \pm 0.066$ \\
\hline
\end{tabular}

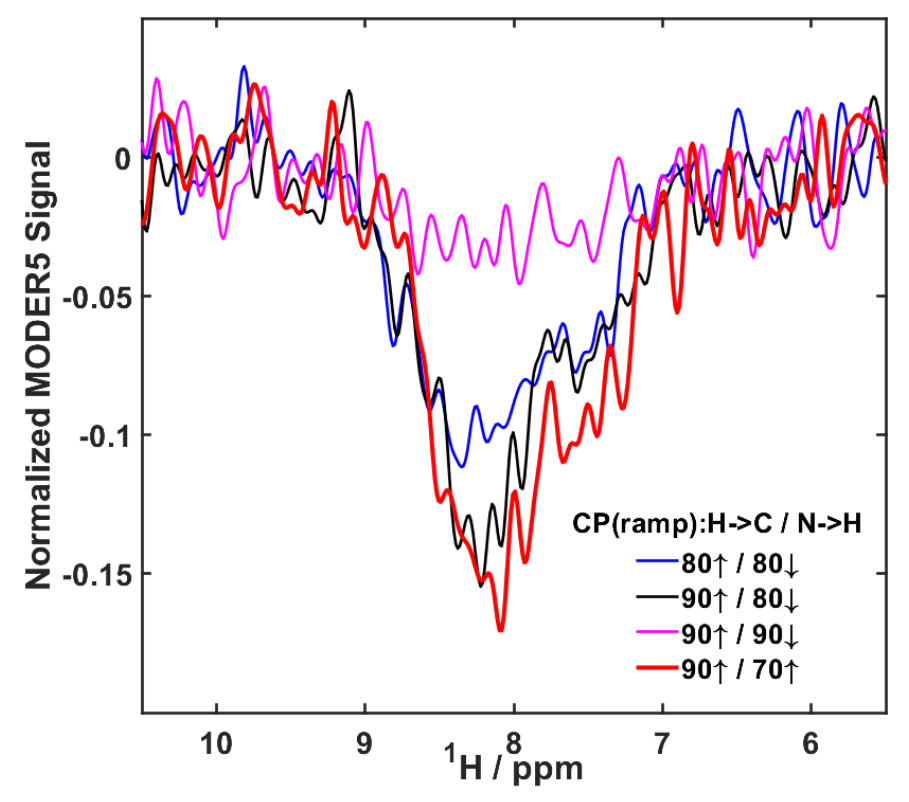

Figure S17. HN MODER5 optimization for M2 S31N at a spinning frequency of $90.908 \mathrm{kHz}$ MAS. MODER5 HN signal (with $154 \mu$ s of MODER5 and optimized power level) for four different sets of ramped CP: blue $-80 \% \rightarrow 100 \%$ for $\mathrm{CH}$ and $100 \% \rightarrow 80 \%$ for $\mathrm{NH}$; black $-90 \% \rightarrow 100 \%$ for $\mathrm{CH}$ and $100 \% \rightarrow 80 \%$ for $\mathrm{NH}$; pink $-90 \% \rightarrow 100 \%$ for $\mathrm{CH}$ and $100 \% \rightarrow 90 \%$ for $\mathrm{NH}$ and red $-90 \% \rightarrow 100 \%$ for $\mathrm{CH}$ and $70 \% \rightarrow 100 \%$ for $\mathrm{NH}$ 
Figures S18-S19 show the experimental and simulated MODER5 curves for M2 (wild type) and M2 (S31N), respectively.

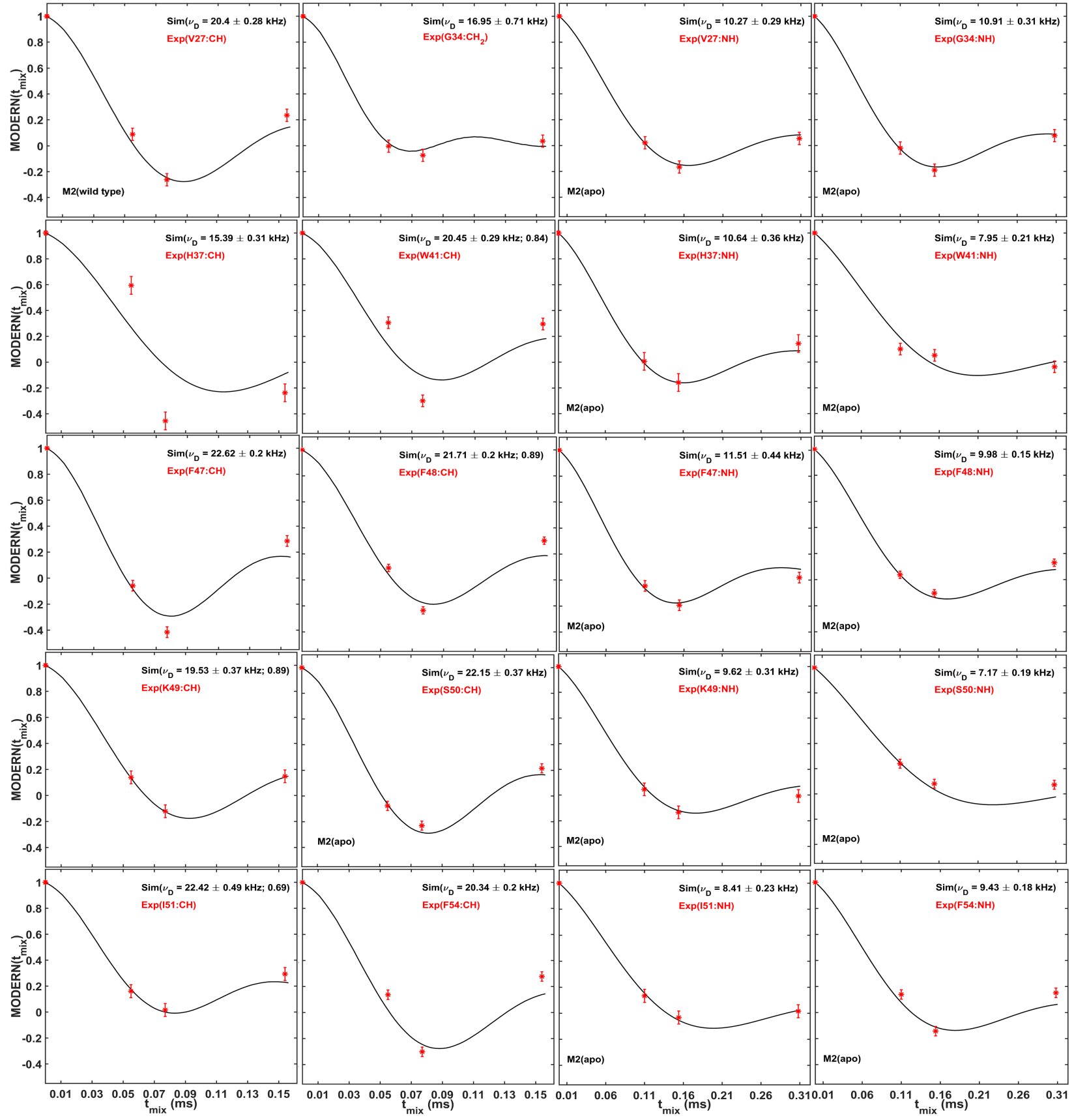

Figure S18. Experimental M2 (wild type) and simulated MODER5 curves for HC (left two columns) and NH (right two columns) dipolar coupling values. The simulated MODER5 curves were obtained with $\Delta \alpha_{r f, i}$ and $T_{2, e f f}$ (Table S2). Eqn. S4 represents the simulated signal. The fitting errors were obtained by generating 100 Monte Carlo curves, assuming a Gaussian noise distribution. Error bars are displayed at 1.5 $\mathrm{\sigma}$. The dataset was recorded as pseudo 3D. 


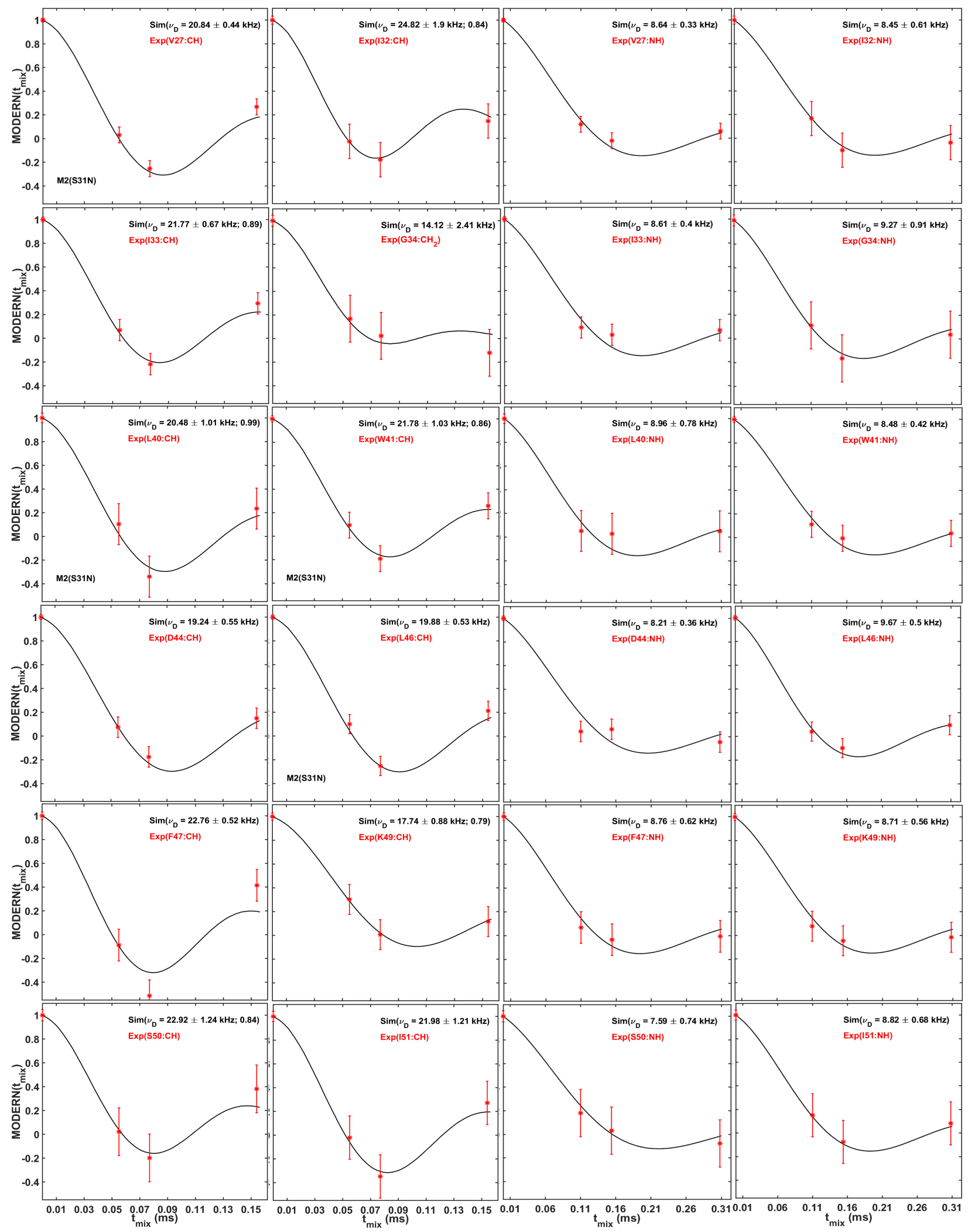

Figure S19. Experimental M2 (S31N) and simulated MODER5 curves for HC (left two columns) and $\mathrm{NH}$ (right two columns) dipolar coupling values. The simulated MODER5 curves were obtained with $\Delta \alpha_{r f, i}$ and $T_{2, e f f}$ according to 
Table S2. Eqn. S4 represents the simulated signal. The fitting errors were obtained by generating 100 Monte Carlo curves, assuming a Gaussian noise distribution. Error bars are displayed at $1.5 \sigma$. The dataset was recorded as pseudo 3D.

Figure S20 summarizes the obtained $\mathrm{HC}(\mathrm{A})$ and $\mathrm{NH}(\mathrm{B})$ dipolar coupling values. Figure $\mathrm{C}$ shows the population of $\mathrm{C}_{\alpha} \mathrm{H}$ pairs with a strongest dipolar coupling. Since this sample did not contain deuteration, the physical interpretation behind this population could be e.g. a small amount of overlapping signal from carbonyl resonances. At faster spinning, CP conditions can be broad enough for some carbonyl signal to survive the CP transfers, despite relatively selective $\mathrm{CP}$ conditions. In the pseudo-3D data, the carbon frequency is not sampled, such that carbonyl signal would overlap.
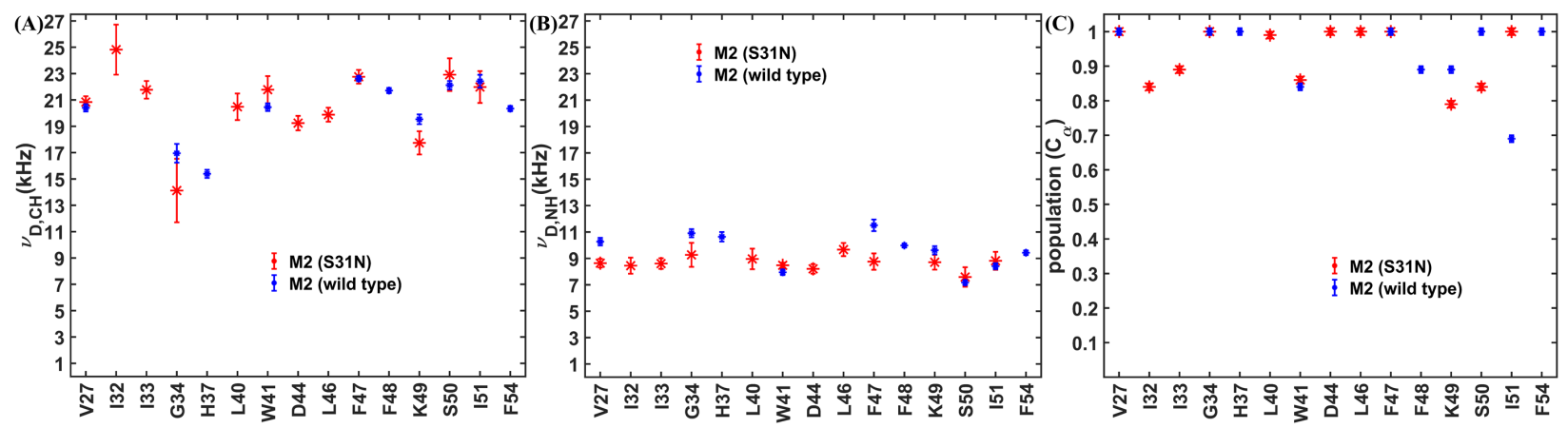

Figure S20. The obtained $\mathrm{HC}(\mathrm{A}), \mathrm{NH}(\mathrm{B})$ dipolar coupling and $\mathrm{C}_{\alpha}$ population $(\mathrm{C})$ values for different M2 samples: S31N - red stars and wild type - blue diamonds.

Figures S21-S22 show the comparison of the experimental and simulated MODER5MODER5 curves for obtaining $\phi_{H}$ values. 

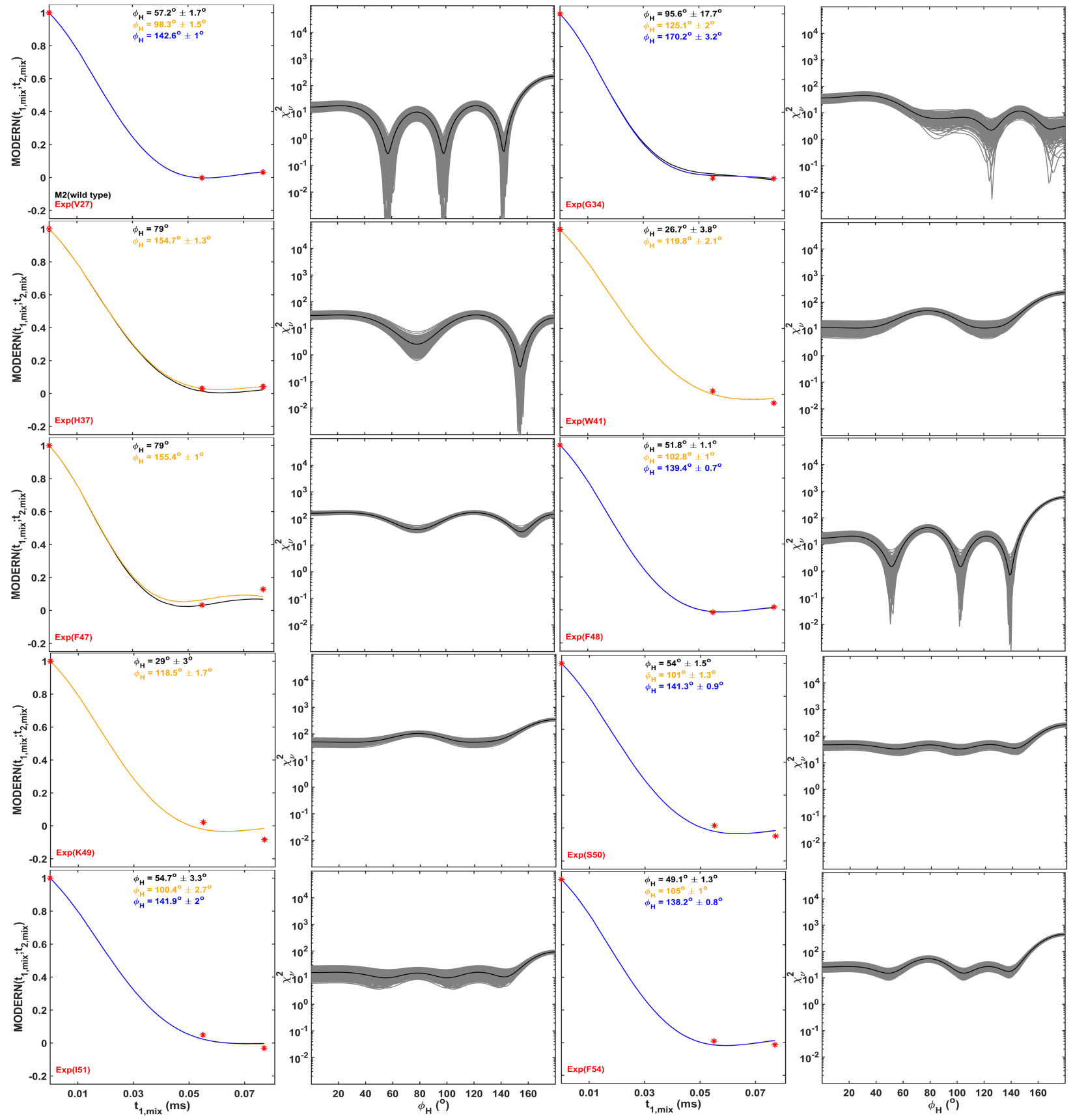

Figure S21. The first and third columns: Experimental M2 (wild type) and simulated MODER5-MODER5 curves (solid lines). The second and fourth columns: $\chi_{v}^{2}$ (Eqn. S1) as a function of torsion angle, $\phi н$. The simulated MODER5MODER5 curves were obtained with $\Delta \alpha_{r f, i}$ and $T_{2, e f f}$ values of Table S2 and measured dipolar coupling values. The fitting errors were obtained by generating 300 Monte Carlo curves (grey curves, the black curve is the average), assuming a Gaussian noise distribution. and considering the error estimate in the determination of dipolar coupling values. Error bars are shown at $1.5 \sigma$. 

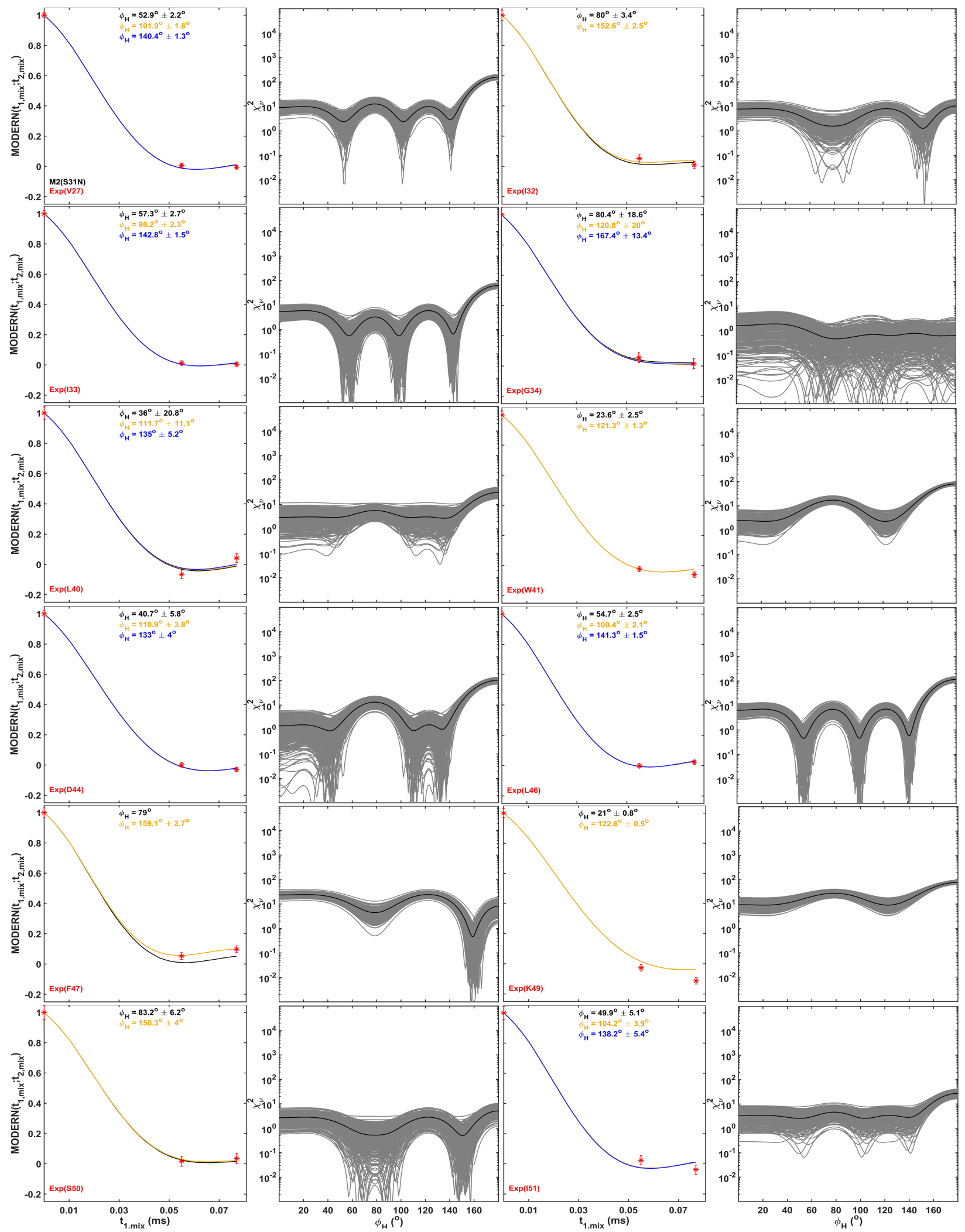

Figure S22. The first and third columns: Experimental M2 (S31N) and simulated MODER5-MODER5 curves (solid lines). The second and fourth columns: $\chi_{v}^{2}$ (Eqn. S1) as a function of torsion angle, $\phi_{\mathrm{H}}$. The simulated MODER5- 
MODER5 curves were obtained with $\Delta \alpha_{r f, i}$ and $T_{2, e f f}$ values of Table S2 and measured dipolar coupling values. The fitting errors were obtained by generating 300 Monte Carlo curves (grey curves, the black curve is the average), assuming a Gaussian noise distribution and considering the errors in the determination of dipolar coupling values. Error bars are shown at $1.5 \sigma$.

Figure S23 compares the obtained $\phi_{\mathrm{H}}$ values for each sample (red stars) with the TALOS predictions (black circles).
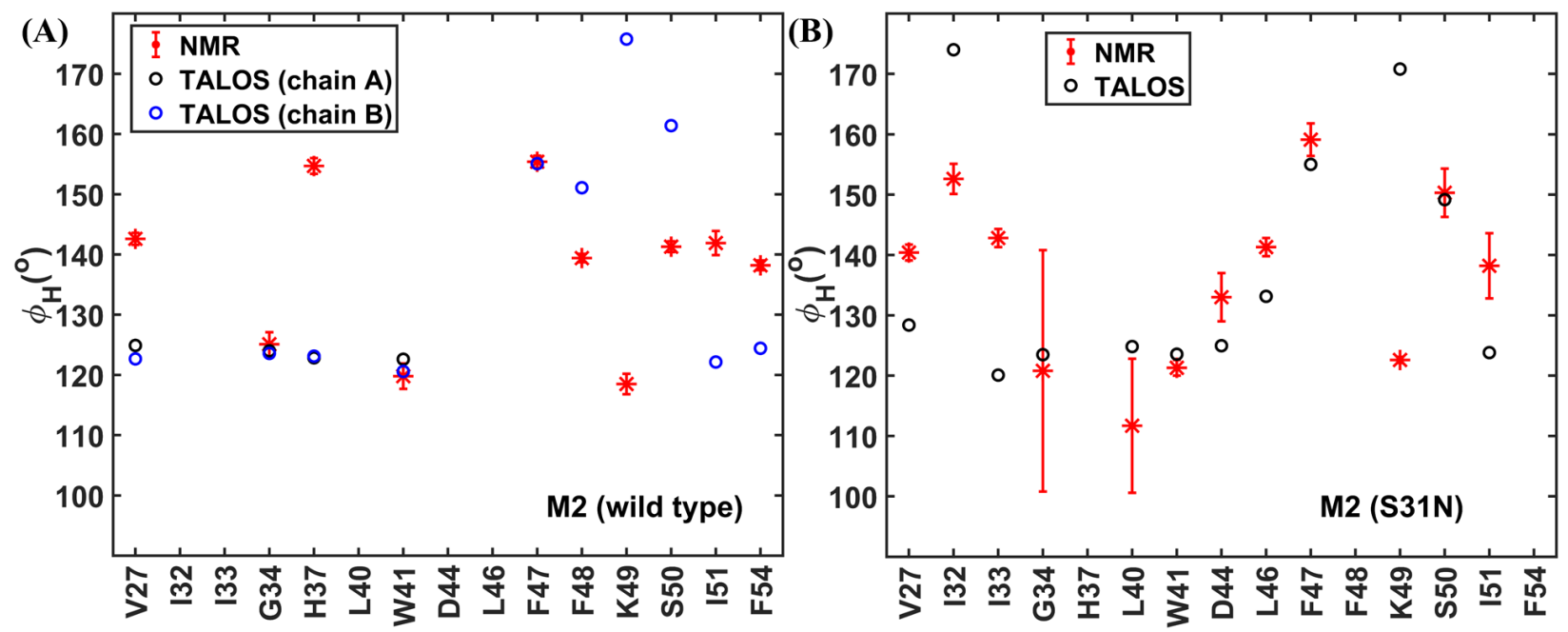

Figure S23. Comparison of the $\phi_{\mathrm{H}}$ values which were obtained with NMR experiments (red stars) and predicted by TALOS (black and blue circles). (A) M2 (wild type) For TALOS calculations the isotropic chemical shift of chains A and $B$ were used. (B) M2 (S31N). For NMR only one set of the possible values were used.

\section{MATLAB Code}

The MATLAB code is divided into three different MATLAB files: 'MODERN_SIMULATIONS.m', 'MODERN_D_Phi.m' and 'MODERN_Plot.m'. All input dipolar coupling values should be in $\mathrm{kHz} /$ the angles in degrees / the time as the number of MODERN blocks $\left(1,2,3, \ldots\right.$ where one MODERN block consists of two $90^{\circ}$ pulses). The first point is always $t_{\mathrm{mix}}=0$.

'MODERN SIMULATIONS.m' allows to simulate MODERN (dipolar coupling experiment, the Flag 'Dipolar_Torsion = 0', line 9) and MODERN-MODERN (torsion angle experiment, the Flag 'Dipolar_Torsion = 1', line 9). The dipolar coupling (D_C, line 11) or torsion angle (Phi_H, line 25) values can be inserted as an array (up to 10). Line 9 determines the increment number of MODERN blocks for the simulation of the dipolar interaction (jump_CH). The dipolar coupling values can be simulated for $\mathrm{CH}, \mathrm{CH} 2$ or $\mathrm{CH} 3$ groups (the flag ' $\mathrm{CH}$ _flag_C', line 14). For the simulation of the torsion angles, the angle can be determined for $\mathrm{HC}-\mathrm{NH}\left(\mathrm{CH} \mathrm{Clag}_{-} \mathrm{C}=1\right.$, $\mathrm{CH}_{-}$flag_N $=1$, lines 14 and 24); $\mathrm{H} 2 \mathrm{C}-\mathrm{NH}\left(\mathrm{CH}\right.$ _flag_C=2, $\left.\mathrm{CH} \_f l a g \_\mathrm{N}=1\right)$ or $\mathrm{H} 2 \mathrm{C}-\mathrm{CH} 2$ $\left(\mathrm{CH}\right.$ _flag_C=2, $\left.\mathrm{CH} \_f l a g \_\mathrm{N}=2\right)$. The MODERN curves can be simulated as ideal (da_rf_distr = 0 , line 17) or with the influence of flip angle distribution and the effective relaxation: da_rf_distr = 1, line 17. The values da_rf_max (line 18) and T2_eff (line 19) are changed manually. The dependence of MODERN pulses can be simulated in the projection angle domain (The flag 
'projection_torsion_domain $=0$ ', line 26) or in the torsion angle domain (The flag 'projection_torsion_domain $=1$ ', line 26). For the torsion angle domain, two additional angles are used: theta_NH (line 31) and theta_CH (line 32).

'MODERN D Phi.m' code allows to obtain the dipolar coupling (The Flag 'Dipolar_Torsion = 0', line 11) and the torsion angle (The Flag 'Dipolar_Torsion = 1', line 11).

'MODERN Plot.m' code allows to plot the experimental and simulated signals with obtained dipolar couplings (The Flag 'Dipolar_Torsion $=0$ ', line 8) and torsion angles (The Flag 'Dipolar_Torsion = 1', line 8).

\section{'MODERN_D_Phi.m' code}

To obtain dipolar coupling values without $\Delta \alpha_{r f, \max }$ and $T_{2, \text { eff }}$ optimization:

'Optimization_rf_T2=0' (Flag at line 14)

'Dipolar_Torsion $=0$ ' (Flag at line 25)

'Exp_D.dat' file should be prepared (line 36). This file can contain from 3 to 5 columns. The first column contains the numbers of applied MODERN blocks (minimal unit is 1 , which defines a length corresponding to two $90^{\circ}$-pulses). The second column contains the experimental signal intensities. The third column contains the experimental errors. If some carbons have more than one directly bonded protons ( $\mathrm{CH} 2$ or $\mathrm{CH} 3$ groups), the fourth column is added. For $\mathrm{CH}, \mathrm{CH} 2$ and $\mathrm{CH} 3$ groups the numbers 1,2 and 3 are added in this column, respectively. If some carbons are partially deuterated, the fifth column is added. For fully protonated cases, 1 is used, whereas for partially deuterated, 0.5. For example, 'Exp_D_aPET_long_CP.dat' file contains all five columns with $\mathrm{CH}, \mathrm{NH}$ experimental data for V9. 'Exp_D_aPET_short_CP.dat' file contains only three columns. The 'Exp_D_SH3_long_CP.dat' file contains four, where $\mathrm{CH} 2$ and $\mathrm{NH}$ of $\mathrm{G} 28$ experimental signals are analyzed. The output file, 'Dipolar values.dat' contains the obtained dipole coupling values (the first row), and associated errors (the second row). For calculations of the dipolar coupling errors, the Monte Carlo curves are generated (The flag 'montynumber $=100$ ', line 23). If the sample is partially deuterated, the third column represents the proton population obtained in the fit. For example, 'Dipolar values.dat' file contains the obtained $\mathrm{CH}$ dipolar coupling value of V9 residue and its population of the signal (0.79). As an example, Table S2 shows the input file for calculation of dipolar couplings, considering different populations and directly bonded protons. Two residues are shown, and the program recognizes the next residue by the 0 in the 'Number of MODERN blocks' column. . 
Table S2. Example of the input file for dipolar coupling determination, consisting of 5 (maximal) columns. The first, second and third columns are the main columns - number of applied MODERN blocks, the experimental signal amplitudes and the errors. The fourth column defines the number of bonded protons (from 1 to 3 ). The fifth column defines the full protonation (1) or partial protonation (0.5). MODER5 $\mathrm{CHn}$ experimental data of two residues (G34 SH3 and $\mathrm{V} 9 \alpha$-PET SH3 with long CP) are shown.

\begin{tabular}{|l|l|l|l|l|}
\hline $\begin{array}{l}\text { Number of } \\
\text { MODERN blocks }\end{array}$ & $\begin{array}{l}\text { Experimental } \\
\text { signal }\end{array}$ & $\begin{array}{l}\text { Experimental } \\
\text { error }\end{array}$ & $\begin{array}{l}\text { Number } \\
\text { bonded protons }\end{array}$ & Population \\
\hline 0 & 5015.706 & 306.2523 & 2 & 1 \\
\hline 6 & 2787.737 & 306.2523 & 2 & 1 \\
\hline 17 & -347.9873 & 306.2523 & 2 & 1 \\
\hline 20 & -822.5881 & 306.2523 & 2 & 1 \\
\hline 32 & -240.2521 & 306.2523 & 2 & 1 \\
\hline 35 & -253.6302 & 306.2523 & 2 & 1 \\
\hline 38 & 4.860873 & 306.2523 & 2 & 1 \\
\hline 0 & 20667.95 & 296.603 & 1 & 0.5 \\
\hline 6 & 13253.89 & 296.603 & 1 & 0.5 \\
\hline 17 & -789.4179 & 296.603 & 1 & 0.5 \\
\hline 20 & -2559.028 & 296.603 & 1 & 0.5 \\
\hline 32 & 6071.143 & 296.603 & 1 & 0.5 \\
\hline 35 & 7440.854 & 296.603 & 1 & 0.5 \\
\hline 38 & 9266.049 & 296.603 & 1 & 0.5 \\
\hline
\end{tabular}

To obtain dipolar coupling values with optimized $\Delta \alpha_{r f, \max }$ and $T_{2, \text { eff }}$ :

First Step

'Optimization_rf_T2=1' (Flag at line 25)

'Dipolar_Torsion $=0$ ' (Flag at line 10)

The file 'T2_RF_OPT_EXP.dat' (line 44) should be prepared. It can contain any number of experimental signals. For example, 'T2_RF_OPT_EXP_aPET_short_CP.dat' file contains the NH experimental curves of four residues of a-PET SH3 (short CP): V9, Y13, A56 and L61. These experimental curves were chosen, since they had the longest MODER5 mixing times (for $\mathrm{NH}$ groups, the MODERN mixing time was two times longer than for $\mathrm{CH}$ group) and the normalized largest amplitudes were observed. The output file, 'opt_var.dat' contains the $\Delta \alpha_{r f, \max }$ with the 
error (the first row) and $T_{2, e f f}$ (the second row). The example of output 'opt_var.dat' file for aPET SH3 (long CP).

As an example, Table S3 shows an example input file for dipole coupling determination with optimization of flip angle distribution and $T_{2, e f f}$.

Table S3. Example input file for the dipolar coupling values determination, consisting of 3 columns. The first, second and third columns are number of the applied MODERN blocks, the experimental signals and the errors. MODER5 NH experimental data of two residues (V9 and Y13 $\alpha$-PET SH3 with short CP) are shown.

\begin{tabular}{|l|l|l|}
\hline Number of MODERN blocks & Experimental signal & Experimental error \\
\hline 0 & 10636.37 & 279.719 \\
\hline 12 & 7921.961 & 279.719 \\
\hline 34 & -2294.437 & 279.719 \\
\hline 40 & -3159.823 & 279.719 \\
\hline 64 & 105.1874 & 279.719 \\
\hline 70 & 1104.539 & 279.719 \\
\hline 76 & 1803.188 & 279.719 \\
\hline 0 & 19682.84 & 279.719 \\
\hline 12 & 14210.3 & 279.719 \\
\hline 34 & -4998.771 & 279.719 \\
\hline 40 & -6647.696 & 279.719 \\
\hline 64 & 2073.059 & 279.719 \\
\hline 70 & 4034.151 & 279.719 \\
\hline 76 & 5297.883 & 279.719 \\
\hline
\end{tabular}

Table S4 shows the example of the output file with the optimal values.

Table S4. The example of the output file with optimal $\Delta \alpha_{r f, \max }$ (the first row) and $T_{2, e f f}$ (the second row) with the errors.

\begin{tabular}{|l|l|l|}
\hline parameter & mean & std \\
\hline \multicolumn{1}{|c|}{$\Delta \alpha_{r f, \max }$} & 0.028 & 0 \\
\hline$T_{2, \text { eff }}(\mathrm{ms})$ & 0.648 & 0.233 \\
\hline
\end{tabular}

\section{Second Step}

'Optimization_rf_T2=2' (Flag at line 25)

'Dipolar_Torsion = 0' (Flag at line 10)

'Exp_D.dat' file should be prepared in the same way as was described above. 
In both cases, the output file, 'Dipolar_values.dat' file can contain two to three columns. The first two columns are the obtained dipolar coupling values and the errors. The third column represents the value of the protonation obtained in the fit $(p \leq 1)$.

To obtain torsion angle values without $\Delta \alpha_{r f, \max }$ and $T_{2, \text { eff }}$ optimization:

'Optimization_rf_T2=0' (Flag at line 25)

Dipolar_Torsion $=1$ ' (Flag at line 10)

The file 'Exp_Phi.dat' (line 48) should be prepared. It can contain from four to six columns. The first two columns define MODERN mixing times (number of blocks) for $\mathrm{CH}$ and $\mathrm{NH}$ groups, respectively. The third and fourth columns are experimental points and errors. The fifth column is added when some carbons have more than one directly bonded protons. The sixth column is added when the other spin can also have more than one directly bonded protons (for example, CH2-CH2). For example, 'Exp_Phi_SH3_long_CP.dat' file contains five columns for V9 and G28 residues, while 'Exp_Phi_aPET_long_CP.dat' file contains four columns. Additionally, the file “Dipolar_values_Phi.dat' should be prepared. The first two columns should contain the obtained $\mathrm{CH}$ dipolar coupling values and their errors. The third and fourth columns should contain the obtained $\mathrm{NH}$ dipolar coupling values and their errors. The fifth column should be if some groups are partially deuterated. For example, 'Dipolar_values_Phi_SH3_long_CP.dat' file contains four columns, while 'Dipolar_values_Phi_aPET_long_CP.dat' file contains five columns. The comparison of the experimental and simulated data can be done with the projection angles (The flag 'projection_torsion_domain = 0', line 14) or in the torsion angle domain (The flag 'projection_torsion_domain =1', line 14). For the torsion angle domain, two additional angles are used: theta_NH (line 19) and theta_CH (line 20).

The output files are two files. In the first, 'phi_best.txt' file, each row contains the obtained torsion angles with the errors. For calculations of the torsion angle errors, the Monte Carlo curves are generated (The flag 'montynumber = 100', line 23) and the errors of the dipolar coupling values are taken into account (three sets of the dipolar coupling values are simulated, the fit value and the fit value \pm the estimated error at 1 standard deviation). The file, "aver_chi_curve.txt' contains the averaged $\chi_{v}^{2}$ over $3^{\star}$ montynumber curves (montynumber - number of Monte Carlo curves, line 23) as the rows, whereas the file, "chi_curve_all.txt" contains all Monte Carlo curves as the rows.

\section{'MODERN_Plot.m' code}

To obtain dipolar coupling plots without $\Delta \alpha_{r f, \max }$ and $T_{2, \text { eff }}$ optimization: 
'Optimization_rf_T2=0' (Flag at line 21)

Dipolar_Torsion $=0$ ' (Flag at line 9)

Additionally to the previous files, one more file should be created - 'Names.dat' (line 27). For example, 'Names_aPET_long_CP.dat' contains the names of V9:CH and V9: $\mathrm{NH}$.

To obtain dipolar coupling plots with $\Delta \alpha_{r f, \max }$ and $T_{2, \text { eff }}$ optimization:

'Optimization_rf_T2=1' (Flag at line 21)

Dipolar_Torsion $=0^{\prime}$ (Flag at line 9)

The same file with the names of the residues should be prepared.

To obtain torsion angle plots without $\Delta \alpha_{r f, \max }$ and $T_{2, \text { eff }}$ optimization:

'Optimization_rf_T2=0' (Flag at line 21)

'Dipolar_Torsion=1' (Flag at line 9)

Additionally to the previous files, one more file should be created - 'Names_Phi.dat' (line 27).

For example, 'Names_Phi.dat' contains the names of V9 and G28.

To obtain dipolar coupling plots with $\Delta \alpha_{r f, \max }$ and $T_{2, \text { eff }}$ optimization:

'Optimization_rf_T2=1' (Flag at line 21)

'Dipolar_Torsion $=1$ ' (Flag at line 9)

The same file with the names of the residues should be prepared.

Table S2 summarizes the run time of the code per one experimental curve with specific flags, when full protonation is assumed. For partial deuteration, the time is increased about 10-fold (for 'Dipolar_Torsion' $=0$ flag only). If the flip angle distribution is larger than 0.1 , all run times are increased two-fold, since 14 MODERN curves are simulated and not 7 .

Table S2 The time required to compare the simulated and the experimental MODERN signals and obtain dipolar coupling or torsion angle values. The calculations were performed on a desktop computer with an Intel® Core ${ }^{\mathrm{TM}}$ i57600 CPU @ 3.5GHz.

\begin{tabular}{|l|l|l|l|l|l|}
\hline Optimization_ff_T2 & 0 & 1 & 2 \\
\hline Dipolar_Torsion & 0 & 1 & --- & 0 & 1 \\
\hline Time (s) & $\sim 10$ & $\sim 140$ & $\sim 100$ & $\sim 70$ & $\sim 1200$ \\
\hline
\end{tabular}

Additionally to the mentioned input files (as examples), we provide a set of the input files, which can be used for running the codes (the experimental data of a-PET SH3 (short CP)):

'T2_RF_OPT_EXP.dat' (contains the experimental NH data of V9, Y13, A56 and L61 residues); 'Exp_D.dat' (contains the experimental $\mathrm{CH}$ data of V9, Y13, A56 residues and then the experimental $\mathrm{NH}$ data of $\mathrm{V} 9, \mathrm{Y} 13, \mathrm{~A} 56$ residues);

'Exp_Phi.dat' (contains the experimental CH-NH data of V9, Y13, A56 residues); 
'NAMES.dat' (contains the names of the residues for dipolar coupling value plots);

'NAMES_Phi.dat' (contains the names of the residues for torsion angle plots).

\section{Sample preparation}

$\alpha$-PET SH3 and fully protonated SH3 samples were prepared according to the protocols in Refs. [-11] and influenza A M2 protein, residues 18-60, was prepared according to the protocols in Refs. $\left[{ }^{12,13}\right]$.

\section{NMR Measurements}

For NMR measurements at $600 \mathrm{MHz}$, either $0.3 \mathrm{~ms}$ (short CP) or $1.5 \mathrm{~ms}$ (long CP) $\mathrm{H}$ to $\mathrm{C}$ was applied, and $5.5 \mathrm{~ms}$ for $\mathrm{N}$ to $\mathrm{C} \mathrm{CP}$ and $0.5 \mathrm{~ms}$ for $\mathrm{N}$ to H CP, in order to limit magnetization transfer to one bond. The MAS was $55 \mathrm{kHz}$ in a $1.3 \mathrm{~mm}$ Bruker $\mathrm{HXY}$ wide-bore probe and the variable temperature (VT) gas was set to $\sim 240 \mathrm{~K}$. For measurements at $800 \mathrm{MHz}$, the short $\mathrm{CP}$ times listed above were used. The MAS was $55 \mathrm{kHz}$ in a Bruker HCN narrow-bore probe and the variable VT gas was set to $\sim 240 \mathrm{~K}$. For measurements at $950 \mathrm{MHz}$, the short CP times listed above were used. The MAS was $90.908 \mathrm{kHz}$ in a Bruker HCND narrow-bore probe and the VT gas was set to $255 \mathrm{~K}$. The $(\mathrm{H}) \mathrm{CANH}$ pulse sequence used as the basis for the torsion angle measurement was described in the publication of the Pintacuda group in 2014 (Ref. ${ }^{14}$ ) and the MODERN pulse sequence is according to Ref. ${ }^{1}$.

1. Nimerovsky, E. \& Soutar, C. P. A modification of $Y$-encoded RN symmetry pulses for increasing the scaling factor and more accurate measurements of the strong heteronuclear dipolar couplings. J. Magn. Reson. 319, 106827 (2020).

2. Ahlner, A., Carlsson, M., Jonsson, B.-H. \& Lundström, P. PINT: a software for integration of peak volumes and extraction of relaxation rates. J. Biomol. NMR 56, 191-202 (2013).

3. Brinkmann, A. \& Levitt, M. H. Symmetry principles in the nuclear magnetic resonance of spinning solids: Heteronuclear recoupling by generalized Hartmann-Hahn sequences. J. Chem. Phys. 115, 357-384 (2001). 
4. Zhao, X., Edén, M. \& Levitt, M. H. Recoupling of heteronuclear dipolar interactions in solidstate NMR using symmetry-based pulse sequences. Chem. Phys. Lett. 342, 353-361 (2001).

5. Paulson, E. K., Martin, R. W. \& Zilm, K. W. Cross polarization, radio frequency field homogeneity, and circuit balancing in high field solid state NMR probes. J. Magn. Reson. 171, 314-323 (2004).

6. Engelke, F. Electromagnetic wave compression and radio frequency homogeneity in NMR solenoidal coils: Computational approach. Concepts Magn. Reson. 15, 129-155 (2002).

7. Gupta, R., Hou, G., Polenova, T. \& Vega, A. J. RF INHOMOGENEITY AND HOW IT CONTROLS CPMAS. Solid State Nucl. Magn. Reson. 72, 17-26 (2015).

8. Xue, K., Mühlbauer, M., Mamone, S., Sarkar, R. \& Reif, B. Accurate Determination of 1H-15N Dipolar Couplings Using Inaccurate Settings of the Magic Angle in Solid-State NMR Spectroscopy. Angew. Chem. Int. Ed. 58, 4286-4290 (2019).

9. Pauli, J., van Rossum, B., Förster, H., de Groot, H. J. M. \& Oschkinat, H. Sample Optimization and Identification of Signal Patterns of Amino Acid Side Chains in 2D RFDR Spectra of the a-Spectrin SH3 Domain. J. Magn. Reson. 143, 411-416 (2000).

10. Chevelkov, V. et al. Differential Line Broadening in MAS Solid-State NMR due to Dynamic Interference. J. Am. Chem. Soc. 129, 10195-10200 (2007).

11. Movellan, K. T. et al. Alpha protons as NMR probes in deuterated proteins. J. Biomol. Nmr 73, 81-91 (2019).

12. Schnell, J. R. \& Chou, J. J. Structure and mechanism of the M2 proton channel of influenza A virus. Nature 451, 591-595 (2008).

13. Andreas, L. B., Eddy, M. T., Pielak, R. M., Chou, J. \& Griffin, R. G. Magic Angle Spinning NMR Investigation of Influenza A M218-60: Support for an Allosteric Mechanism of Inhibition. J. Am. Chem. Soc. 132, 10958-10960 (2010). 
14. Barbet-Massin, E. et al. Rapid Proton-Detected NMR Assignment for Proteins with Fast Magic Angle Spinning. J. Am. Chem. Soc. 136, 12489-12497 (2014). 